Article

\title{
From Relativistic Mechanics towards Relativistic Statistical Mechanics
}

\section{Luca Lusanna}

Retired Research Director of Sezione INFN di Firenze, Via G. Sansone 1, 50019 Sesto Fiorentino (FI), Italy; lusanna@fi.infn.it

Received: 22 May 2017; Accepted: 5 August 2017; Published: 23 August 2017

\begin{abstract}
Till now, kinetic theory and statistical mechanics of either free or interacting point particles were well defined only in non-relativistic inertial frames in the absence of the long-range inertial forces present in accelerated frames. As shown in the introductory review at the relativistic level, only a relativistic kinetic theory of "world-lines" in inertial frames was known till recently due to the problem of the elimination of the relative times. The recent Wigner-covariant formulation of relativistic classical and quantum mechanics of point particles required by the theory of relativistic bound states, with the elimination of the problem of relative times and with a clarification of the notion of the relativistic center of mass, allows one to give a definition of the distribution function of the relativistic micro-canonical ensemble in terms of the generators of the Poincare algebra of a system of interacting particles both in inertial and in non-inertial rest frames. The non-relativistic limit allows one to get the ensemble in non-relativistic non-inertial frames. Assuming the existence of a relativistic Gibbs ensemble, also a "Lorentz-scalar micro-canonical temperature" can be defined. If the forces between the particles are short range in inertial frames, the notion of equilibrium can be extended from them to the non-inertial rest frames, and it is possible to go to the thermodynamic limit and to define a relativistic canonical temperature and a relativistic canonical ensemble. Finally, assuming that a Lorentz-scalar one-particle distribution function can be defined with a statistical average, an indication is given of which are the difficulties in solving the open problem of deriving the relativistic Boltzmann equation with the same methodology used in the non-relativistic case instead of postulating it as is usually done. There are also some comments on how it would be possible to have a hydrodynamical description of the relativistic kinetic theory of an isolated fluid in local equilibrium by means of an effective relativistic dissipative fluid described in the Wigner-covariant framework.
\end{abstract}

Keywords: special relativity; statistical mechanics; kinetic theory

\section{Introduction}

In non-relativistic physics, the description of systems of interacting $\mathrm{N}$ point particles in the Euclidean three-spaces of the inertial frames of Galilei space-time can be done without problems due to the existence of an absolute time. The non-relativistic center of mass and the generators of the Galilei group are well defined. As a consequence, there is a well-defined formulation of kinetic theory and statistical mechanics in non-relativistic inertial frames [1,2]. However, the extension to non-inertial frames is mainly restricted to the region near the axis of rotating frames without a universally-accepted formulation in arbitrarily-moving frames, where the inertial forces are always long-range forces.

In special relativity, free point particles follow world-lines described by four-vector configuration variables $q_{i}^{\mu}\left(\tau_{i}\right), i=1, \ldots, N$, each one depending on a proper time $\tau_{i}$ (see Endnote [3]—which refers to Reference [4]). At the Hamiltonian level, the canonical four-vector position variables $x_{i}^{\mu}(\tau)$ are all functions of the unique proper time $\tau$ of a given observer and are supposed to coincide with the configuration ones: $x_{i}^{\mu}(\tau)=q_{i}^{\mu}\left(\tau_{i}=\tau\right), i=1, \ldots, N$. Moreover, the conjugate momenta $p_{i \mu}(\tau)$ 
must satisfy a mass-shell constraint, whose form is $p_{i \mu} p_{i}^{\mu}=m_{i}^{2} c^{2}$ in the absence of interactions. As a consequence, the energies $p_{i o}(\tau)$ are not independent variables, and the time components $x_{i}^{o}(\tau)$ are gauge variables according to the Dirac theory of constraints [5]. To describe either action-at-a-distance interactions or the coupling to the electro-magnetic field to the end of studying relativistic bound states (already at the classical level before quantization), one needs to introduce some type of synchronization to eliminate the relative times, because there is no trace of time-like excitations in the particle spectra. After more than thirty years of efforts to solve these problems in 1965, Currie, Jordan and Sudarshan [6] demonstrated a no-interaction theorem according to which in the presence of a canonical realization of the Poincaré, group one can have $q_{i}^{\mu}(\tau)=x_{i}^{\mu}(\tau)$ with these quantities being four-vectors only in the free case. In the next forty years, many different approaches trying to overcome the theorem were developed: see $[7,8]$ for a review of all of these attempts.

As a consequence, it was not possible to define a consistent relativistic kinetic theory and then relativistic statistical mechanics in the inertial frames of Minkowski space-time. Therefore, in [9], after a review of the quoted problems, Steward said that it is only possible to define a relativistic kinetic theory of world-lines, but not of relativistic particles. All of the existing approaches [2,10-17] (see also the review in [18]) either consider only free particles or make ad hoc ansatzs whose validity is out of control.

Finally, in [19] was the definition of a general scheme for defining a mathematical method of synchronization, the so-called $3+1$ splitting of Minkowski space-time, allowing one to describe particles, fluids, fields and every system admitting a Lagrangian description in both global inertial and not inertial frames in Minkowski space-time with the transition from one frame to another one described as a gauge transformation by means of the so-called parametrized Minkowski theories. This allowed putting under control the problem of the relativistic center of mass [20-24], to define Wigner-covariant relative three-variables in the instantaneous three-spaces of the inertial rest frame, to study the electro-magnetic field in this framework $[25,26]$ and to define the classical basis of relativistic atomic physics [27-30]. Finally, a consistent relativistic Wigner-covariant quantum mechanics of point particles was defined in [31] after previous attempts [32,33] of defining it in non-inertial frames.

This approach allowed one for the first time to define the relativistic micro-canonical ensemble in the Hamiltonian framework for systems of $\mathrm{N}$ particles interacting through either short- or long-range forces both in inertial frames and in a special family of non-inertial ones (the non-inertial rest frames) of Minkowski space-time [34] (see [35] for an extended version with extra results, which will be used in this paper) and a Lorentz scalar micro-canonical temperature $T_{(m c)}$ (see Endnote [36] - which refers to References [37-42]) extending the non-relativistic results of [43] for the extended distribution function of the micro-canonical ensemble (see Endnote [44]—which refers to Reference [45]) in the presence of long-range forces (see Endnote [46]—which refers to References [47-50]). When the forces in inertial frames are short range and the thermodynamical limit $(N, V \rightarrow \infty$ with $N / V=$ const.) exists, one can define the relativistic canonical ensemble with a Lorentz scalar temperature (see Endnote [51]—which refers to References [52-61]). Furthermore, the Liouville operators for single particles and the one-particle distribution function in relativistic kinetic theory can be defined in the relativistic frames of the $3+1$ approach.

Due to the non-covariance of the canonical relativistic center of mass, one must decouple it, and one must reformulate the dynamics in the relativistic inertial frames only in terms of canonical Wigner-covariant relative variables $\vec{\rho}_{a}(\tau), \vec{\pi}_{a}(\tau), a=1, \ldots, N-1$. As a consequence, the distribution function of the relativistic micro-canonical ensemble is defined only after the decoupling of the center of mass and depends on the ten so-called internal Poincare generators, which are functions only of the relative variables. In relativistic non-inertial frames, the explicit form of the relative variables is not known, but action-at-a-distance potentials can be described by using the Synge world-function, like in general relativity [62]. As a consequence, the explicit form of the micro-canonical distribution in arbitrary non-inertial frames is not known.

In the non-relativistic limit, it is possible to get the (ordinary and extended) Newtonian micro-canonical ensembles both in inertial (see Endnote [63]—which refers to Reference [64]) and non-inertial rest frames 
of Galilei space-time. They are functions of the generators of the Galilei group without a dependence on the center of mass in the Hamilton-Jacobi framework. However, now, one can reintroduce the motion of the center of mass and recover the known definition of the distribution function.

Since in the non-inertial rest frames, both the Galilei or Poincaré generators are asymptotic constants of motion at spatial infinity, also in them, the micro-canonical distribution function is time independent, like in the inertial frames: therefore, the standard passive viewpoint (see Endnote [65]—which refers to Reference [66]) on equilibrium in inertial frames can be extended also to non-inertial rest frames, notwithstanding the fact that the inertial forces are long range independent of the type of inter-particle interactions.

Let us remark that to our knowledge, this is the first time that one has a definition of the micro-canonical ensemble in the rest frame family of relativistic and non-relativistic non-inertial frames.

See [67] for a rich bibliography for the use of these notions in astrophysics, cosmology, Brownian motion, plasma physics, heavy ion collisions and quark-gluon plasma. The presence in this framework of the electro-magnetic field in the radiation gauge is described in $[25,26,68,69]$, but the consequences of its presence in non-inertial frames for plasma physics (Vlasov equation) and magneto-hydrodynamics have still to be explored. See [70-73] for attempts to describe various types of matter in non-inertial frames.

I will review what information and techniques from special relativity (like clock synchronization) have to be used to arrive at the definition a consistent relativistic micro-canonical ensemble. Then, I will introduce relativistic one-particle distribution functions, and I will indicate the problems to be faced to arrive at a relativistic Boltzmann equation by using the same tools needed for its definition in the non-relativistic case. This paper is an attempt to find a description unifying the formalisms of special relativity and statistical mechanics so as to open the path to face more basic physical problems in equilibrium and non-equilibrium relativistic physics.

In Section 2 is the description of the Wigner-covariant relativistic classical mechanics (RCM) for isolated systems of [31] in inertial frames in the framework of the $3+1$ splitting method and of parametrized Minkowski theories with a discussion of the problem of the relativistic center of mass. In Section 2.1 is the definition of the inertial rest frame, whose non-relativistic limit is shown in Section 2.2.

In Section 3 is the formulation of RCM in non-inertial global frames and the definition of the non-inertial rest frames of isolated systems, whose non-relativistic limit is shown in Section 3.1.

In Section 4 is a review of the micro-canonical ensemble for an isolated system of particles in non-relativistic inertial frames. In Section 4.1, it is defined in the non-relativistic inertial rest frame, and then in Section 4.2, it is defined in the relativistic rest frame in Minkowski space-time.

In Section 5, the micro-canonical ensemble is studied in the family of non-inertial rest frames: in Section 5.1 in the relativistic rest frames and in Section 5.2 in the non-relativistic ones.

In Section 6 is the definition of the Lorentz scalar micro-canonical temperature.

In Section 7, after the definition of the non-relativistic one-particle distribution, is a description of the Boltzmann equation and of its postulated extensions. In Section 7.1 is the definition of the relativistic one-particle distribution function in the relativistic inertial rest frames. In Section 7.2 is the indication of the steps necessary to arrive at the relativistic Boltzmann equation with the same methodology of the non-relativistic case.

In Section 8 is a review of the papers concerning a hydrodynamical description of relativistic kinetic theory by means of an effective dissipative fluid. In Section 8.1 are the notations used for such a description, while in Section 8.2 is a sketch of how this description can be formulated in terms of relativistic dissipative fluids described by an action principle in the relativistic inertial rest frame.

In Section 9 are some conclusions. 


\section{Relativistic Mechanics of $\mathrm{N}$ Interacting Particles in Inertial Frames and Its Non-Relativistic Limit}

In $[25,26]$ is the formulation of a theory of non-inertial frames in Minkowski space-time [74] based on a $3+1$ splitting of the space-time, namely on a mathematical idealization of a protocol for clock synchronization:

(a) one gives an arbitrary time-like observer endowed with an atomic clock, and one parametrizes the time-like observer world-line $x^{\mu}(\tau)$ with a time parameter $\tau$, which is an arbitrary monotonically increasing function of the proper time of the clock;

(b) then, one makes an arbitrary nice foliation of Minkowski space-time with space-like instantaneous three-spaces $\Sigma_{\tau}$ (in general, they are not Euclidean three-spaces; they are Euclidean only when the foliation defines an inertial frame) with all of the clocks in the three-space synchronized to the value $\tau$ of the clock of the observer at the intersection of $\Sigma_{\tau}$ with the world-line;

(c) each admissible foliation has the three-spaces tending in a direction-independent way to parallel space-like hyper-planes (i.e., there is an asymptotic inertial frame at spatial infinity);

(d) curvilinear three-coordinates $\sigma^{r}, r=1,2,3$, having the observer as the origin, are defined in each three-space $\Sigma_{\tau}$.

The four-coordinates $\sigma^{A}=\left(\tau ; \sigma^{r}\right)$ are named radar four-coordinates: they were introduced by Bondi [75] in general relativity and have been used for the definition of global non-inertial frames by means of $3+1$ splittings of a certain family of Einstein space-times (see [76-81] and the reviews [82,83]).

The change of four-coordinates $x^{\mu} \mapsto \sigma^{A}\left(x^{\mu}\right)$ from the Cartesian four-coordinates of an inertial observer to the radar four-coordinates of an arbitrary (either inertial or non-inertial) observer have an inverse $\sigma^{A} \mapsto x^{\mu}=z^{\mu}\left(\tau, \sigma^{r}\right)$ with the functions $z^{\mu}\left(\tau, \sigma^{r}\right)$ describing the embedding of the three-spaces $\Sigma_{\tau}$ into Minkowski space-time. By means of the embeddings and of their gradients $z_{A}^{\mu}\left(\tau, \sigma^{r}\right)=\partial z^{\mu}\left(\tau, \sigma^{r}\right) / \partial \sigma^{A}$ [84], one can define the induced four-metric ${ }^{4} g_{A B}\left(\tau, \sigma^{r}\right)=z_{A}^{\mu}\left(\tau, \sigma^{r}\right) \eta_{\mu \nu} z_{B}^{\nu}\left(\tau, \sigma^{r}\right)$ on the three-spaces $\Sigma_{\tau}$ [85]. With radar four-coordinates, both the induced four-metric ${ }^{4} g_{A B}\left(\tau, \sigma^{r}\right)$ and every tensor $A_{A B}\left(\tau, \sigma^{r}\right)=z_{A}^{\mu}\left(\tau, \sigma^{r}\right) A_{\mu v} z_{B}^{v}\left(\tau, \sigma^{r}\right)$ have all of the components, which are Lorentz scalars $[77,78]$.

Every isolated system admitting a Lagrangian description can be described in an arbitrary non-inertial frame with an action principle containing also the embeddings $z^{\mu}(\tau, \vec{\sigma})$ as Lagrangian variables by using the parametrized Minkowski theories defined in $[25,26]$. This is obtained by coupling the Lagrangian of the isolated system to an external gravitational field and by replacing its four-metric with the induced four-metric ${ }^{4} g_{A B}\left(\tau, \sigma^{r}\right)=z_{A}^{\mu}\left(\tau, \sigma^{r}\right) \eta_{\mu v} z_{B}^{v}\left(\tau, \sigma^{r}\right)$. In this formulation, the transition from a frame to any another one is a gauge transformation, because the embeddings $z^{\mu}(\tau, \vec{\sigma})$ turn out to be gauge variables. This is due to the fact that the action principle is invariant under frame-preserving diffeomorphisms, so that in the Hamiltonian formulation, the conjugate canonical momenta $\rho_{\mu}(\tau, \vec{\sigma})$ of the embeddings are determined by four first-class constraints $\rho_{\mu}(\tau, \vec{\sigma}) \approx\left(\sqrt{-g} z_{A \mu} T^{\tau A}\right)(\tau, \vec{\sigma})$ in terms of the embeddings and of the energy-momentum tensor $T^{\mu v}(z(\tau, \vec{\sigma}))=\left(z_{A}^{\mu} z_{B}^{v} T^{A B}\right)(\tau, \vec{\sigma})=$ $l^{\mu} l^{v} T_{\perp \perp}+\left(l^{\mu} z_{r}^{v}+l^{v} z_{r}^{\mu}\right) h^{r s} T_{\perp s}+z_{r}^{\mu} z_{s}^{\mu} T^{r s}$ [86] of the isolated system. The ten Poincare generators of the isolated system are [87] $P^{\mu}=\int d^{3} \sigma \rho^{\mu}(\tau, \vec{\sigma})$ and $J^{\mu v}=\int d^{3} \sigma\left(z^{\mu} \rho^{v}-z^{v} \rho^{\mu}\right)(\tau, \vec{\sigma})$.

Each isolated system has extra first-class constraints (like the mass shell conditions for relativistic particles), implying that the time variables are gauge variables, so that the physics depends only on the spatial three-coordinates inside each three-space. Therefore, in special relativity, the Hamiltonian approach with Dirac constraints is the natural formalism to eliminate the problem of relative times.

The next problem is the decoupling of a collective variable, describing the various types of the relativistic center of mass and tending to the Newtonian center of mass in the non-relativistic limit, from suitable relativistic Hamiltonian relative three-variables describing the inner motions of the isolated'system. As shown in [31], the total four-momentum of an isolated system may be written in the form $P^{\mu}=M c h^{\mu}=M c\left(\sqrt{1+\vec{h}^{2}} ; \vec{h}\right)$, where $M c$ is the invariant mass and $\vec{h}$ is the three-velocity of the 
system. If $\vec{h}$ is considered as a momentum variable, then the conjugate position variable can be shown to be an external decoupled (non-local, non-measurable) non-covariant frozen (i.e., non evolving) three-center of mass $\vec{z}$ [88]. The quantities $\vec{z}$ and $\vec{h}$ are Jacobi data, which tend to the Hamilton-Jacobi version of the Newton center of mass in the non-relativistic limit. This non-covariant collective variable carries a pole-dipole structure, i.e., the invariant mass $M c$ and the rest spin $\vec{S}$ of the isolated system [89] and an external realization of the Poincaré algebra with generators $P^{\mu}=M c h^{\mu}$, $J^{i j}=z^{i} h^{j}-z^{j} h^{i}+\epsilon^{i j k} S^{k}, K^{i}=J^{o i}=-\sqrt{1+\vec{h}^{2}} z^{i}+\frac{(\vec{S} \times \vec{h})^{i}}{1+\sqrt{1+\vec{h}^{2}}}$.

The invariant mass $\mathrm{M}$ and the rest spin $\vec{S}$ of the isolated system are determined by the energy-momentum tensor of the isolated system, i.e., $M c=\int d^{3} \sigma T^{\tau \tau}(\tau, \vec{\sigma})$, $S^{r}=\frac{1}{2} \delta^{r s} \epsilon_{\text {suv }} \int d^{3} \sigma \sigma^{u} T^{\tau v}(\tau, \vec{\sigma})$ inside the three-spaces of the $3+1$ splitting, where the matter three-variables must be re-expressed in terms of suitable relative three-variables without center of mass degrees of freedom. Mc and $\vec{S}$ are the energy and angular momentum generators of an internal realization of the Poincare algebra together with $\mathcal{P}^{r}=\int d^{3} \sigma T^{\tau r}(\tau, \vec{\sigma})$ and $\mathcal{K}^{r}=-\int d^{3} \sigma \sigma^{r} T^{\tau \tau}(\tau, \vec{\sigma})$.

\subsection{Classical Relativistic Mechanics in the Inertial Rest Frame Instant Form}

In the $3+1$ approach, the inertial frames of Minkowski space-time are described by embeddings $z^{\mu}\left(\tau, \sigma^{r}\right)=x^{\mu}(\tau)+f_{r}^{\mu} \sigma^{r}$ with $f_{r}^{\mu}=$ const. and with $x^{\mu}(\tau)=x_{o}^{\mu}+u^{\mu} \tau$ being the world-line of an inertial observer with constant time-like four-velocity $u^{\mu}$.

However, there is a privileged inertial frame associated with every isolated system: the inertial rest frame centered on the inertial observer defined by the non-canonical covariant external Fokker-Pryce four-center of inertia $Y^{\mu}(\tau)$ of the isolated system [90], whose instantaneous Euclidean three-spaces, named Wigner three-spaces due to the fact that the three-vectors inside them are Wigner spin-1 three-vectors [25,26], are orthogonal to the conserved four-momentum $P^{\mu}$ of the isolated system. Therefore, the rest frame of an isolated system is identified by the three first-class constraints $\mathcal{P}^{r} \approx 0$ (named the rest frame conditions) implying the vanishing of the internal three-momentum. These constraints imply that the internal three-center of mass inside the three-spaces is a gauge variable [91], which can be eliminated $[25,26,31]$ by imposing the vanishing of the internal interaction-dependent Lorentz boosts $\mathcal{K}^{r} \approx 0$. With this gauge fixing, one avoids a double counting of the center of mass (one external and one internal), and one gets an unfaithful realization of the internal Poincaré algebra (only the generators $M$ and $\vec{S}$ are not vanishing). The dynamics of the isolated system has now to be re-expressed in terms of suitable Hamiltonian internal Wigner-covariant relative three-variables.

The form of the embedding of the Wigner three-spaces of the inertial rest frame in Minkowski space-time described in the four-coordinates $x^{\mu}$ of the inertial frame of an arbitrary inertial observer is:

$$
\begin{aligned}
z_{W}^{\mu}(\tau, \vec{\sigma})= & Y^{\mu}(\tau)+\epsilon_{r}^{\mu}(\vec{h}) \sigma^{r}=Y^{\mu}(0)+\Lambda_{A}^{\mu}(\vec{h}) \sigma^{A}, \\
& \epsilon_{\tau}^{\mu}(\vec{h})=\frac{P^{\mu}}{M c}=u^{\mu}(P)=h^{\mu}=\left(\sqrt{1+\vec{h}^{2}} ; \vec{h}\right)=\Lambda_{\tau}^{\mu}(\vec{h}), \quad \epsilon P^{2}=M^{2} c^{2}, \\
& \epsilon_{r}^{\mu}(\vec{h})=\left(h_{r} ; \delta_{r}^{i}+\frac{h^{i} h_{r}}{1+\sqrt{1+\vec{h}^{2}}}\right)=\Lambda_{r}^{\mu}(\vec{h}), \quad \eta_{\mu v} \epsilon_{A}^{\mu}(\vec{h}) \epsilon_{B}^{v}(\vec{h})=\eta_{A B},
\end{aligned}
$$

where the Lorentz matrix $\Lambda^{\mu}{ }_{A}(\vec{h})$ is obtained from the standard Wigner boost $\Lambda^{\mu}{ }_{v}\left(P^{\alpha} / M c\right)$, sending the time-like four-vector $P^{\mu} / M c=h^{\mu}$ into $(1 ; 0)$, by replacing the index $v$ with a radar index $A$.

To develop relativistic kinetic theory, one has to describe in this framework isolated systems of either free or interacting particles: this was done in [24-28,31] (see [8] for a review). In the $3+1$ approach, each scalar positive-energy particle is described by radar three-coordinates $\eta_{i}^{r}(\tau)$ individuating the intersection of the world-line $x_{i}^{\mu}(\tau), i=1, \ldots, N$, with the instantaneous three-space $\Sigma_{\tau}: x_{i}^{\mu}(\tau)=z^{\mu}\left(\tau, \eta_{i}^{r}(\tau)\right)$; and by conjugated three-momenta $\kappa_{i r}(\tau)$ with $\left\{\eta_{i}^{r}(\tau), \kappa_{j}^{s}(\tau)\right\}=\delta_{i j} \delta^{r s}$.

The particle world-lines $x_{i}^{\mu}(\tau)$ and the standard momenta $p_{i \mu}(\tau)$ are derived quantities functions of $\tau$, of the external center of mass non-covariant variables $\vec{z}, \vec{h}$ and of suitable relative 
three-variables $\rho_{a}^{r}(\tau), \pi_{a r}(\tau), a=1, \ldots, N-1$, defined inside the three-spaces $\Sigma_{\tau}$ after the elimination of the internal center of mass; they turn out to be covariant, but not canonical predictive coordinates and momenta. The Lorentz-scalar invariant mass $M$ and the rest spin $\vec{S}$ are functions only of the relative variables, and the Hamiltonian for the evolution in the Wigner three-spaces is Mc.

In the inertial rest frame, the world-lines and the standard momenta of $\mathrm{N}$ free positive-energy spinless particles, parametrized in terms of Wigner three-vectors $\vec{\eta}_{i}(\tau)$ and of their canonically conjugate momenta $\vec{\kappa}_{i}(\tau), i=1, \ldots, N$, have the expression $\left(\eta_{i}^{A}(\tau)=\left(\tau ; \eta_{i}^{r}(\tau)\right)\right)$ :

$$
\begin{aligned}
& x_{i}^{\mu}(\tau)=z_{W}^{\mu}\left(\tau, \vec{\eta}_{i}(\tau)\right)=Y^{\mu}(\tau)+\epsilon_{r}^{\mu}(\vec{h}) \eta_{i}^{r}(\tau)=Y^{\mu}(0)+\Lambda_{A}^{\mu}(\vec{h}) \eta_{i}^{A}(\tau), \\
& p_{i}^{\mu}(\tau)=\Lambda_{A}^{\mu}(\vec{h}) \kappa_{i}^{A}(\tau), \quad \kappa_{i}^{A}(\tau)=\left(E_{i}(\tau) ; \kappa_{i r}(\tau)\right) .
\end{aligned}
$$

For free particles, the mass-shell constraints $\epsilon p_{i}^{2} \approx m_{i}^{2} c^{2}$ imply $E_{i}(\tau) \approx \sqrt{m_{i}^{2} c^{2}+\vec{\kappa}_{i}^{2}(\tau)}$; the Hamiltonian is $H=M c=\sum_{i} E_{i}$, and the internal angular momentum is $\vec{S}=\sum_{i=1}^{2} \vec{\eta}_{i}(\tau) \times \vec{\kappa}_{i}(\tau)$.

In the two-body case (see $[8,24,25,27-29]$ for the $\mathrm{N}$-body case), a canonical transformation allows one to get the following collective (the notation $\vec{\eta}_{+}, \vec{\kappa}_{+}=\overrightarrow{\mathcal{P}} \approx 0$ for the internal three-center of mass inside the Wigner three-space is used) and relative variables $\left(m=m_{1}+m_{2}\right)$ :

$$
\begin{aligned}
& \vec{\eta}_{+}=\frac{m_{1}}{m} \vec{\eta}_{1}+\frac{m_{2}}{m} \vec{\eta}_{2}, \quad \vec{\rho}=\vec{\eta}_{1}-\vec{\eta}_{2}, \\
& \vec{\kappa}_{+}=\vec{\kappa}_{1}+\vec{\kappa}_{2} \approx 0, \quad \vec{\pi}=\frac{m_{2}}{m} \vec{\kappa}_{1}-\frac{m_{1}}{m} \vec{\kappa}_{2},
\end{aligned}
$$

whose inversion gives $\vec{\eta}_{1}=\vec{\eta}_{+}+\frac{m_{2}}{m} \vec{\rho}, \vec{\kappa}_{1}=\frac{m_{1}}{m} \vec{\kappa}_{+}+\vec{\pi} \approx \vec{\pi}, \vec{\eta}_{2}=\vec{\eta}_{+}-\frac{m_{1}}{m} \vec{\rho}$. $\vec{\kappa}_{2}=\frac{m_{2}}{m} \vec{\kappa}_{+}-\vec{\pi} \approx-\vec{\pi}$. The gauge fixing $\overrightarrow{\mathcal{K}} \approx 0$ eliminates the internal three-center of mass: $\vec{\eta}_{+}(\tau) \approx$ $\frac{\frac{m_{1}}{m} \sqrt{m_{2}^{2} c^{2}+\vec{\pi}^{2}(\tau)}-\frac{m_{2}}{m} \sqrt{m_{1}^{2} c^{2}+\vec{\pi}^{2}(\tau)}}{\sqrt{m_{1}^{2} c^{2}+\vec{\pi}^{2}(\tau)}+\sqrt{m_{2}^{2} c^{2}+\vec{\pi}^{2}(\tau)}} \vec{\rho}(\tau)\left(\vec{\eta}_{+}(\tau) \approx 0\right.$ for $\left.m_{1}=m_{2}\right)$. As a consequence, the world-lines have the expression [92] $x_{i}^{\mu}(\tau) \approx Y^{\mu}(\tau)+\epsilon_{r}^{\mu}(\vec{h}) \frac{\sqrt{m_{i}^{2} c^{2}+\vec{\pi}^{2}(\tau)}}{\sqrt{m_{1}^{2} c^{2}+\vec{\pi}^{2}(\tau)}+\sqrt{m_{2}^{2} c^{2}+\vec{\pi}^{2}(\tau)}} \rho^{r}(\tau)$, while the standard momenta are $p_{1}^{\mu}(\tau) \approx h^{\mu} \sqrt{m_{1}^{2} c^{2}+\vec{\pi}^{2}(\tau)}+\epsilon_{r}^{\mu}(\vec{h}) \pi^{r}(\tau), p_{2}^{\mu}(\tau) \approx h^{\mu} \sqrt{m_{2}^{2} c^{2}+\vec{\pi}^{2}(\tau)}-\epsilon_{r}^{\mu}(\vec{h}) \pi^{r}(\tau)$.

See [22-25,27] and Section IIB of [35] for the definition of the relative variables of $N$ free particles.

In the interacting case, it is $E_{i} \neq \sqrt{m_{i}^{2} c^{2}+\vec{\kappa}_{i}^{2}(\tau)}$, because the mass-shell conditions become interaction dependent, namely $\epsilon p_{i}^{2} \neq m_{i}^{2} c^{2}$. Moreover, the gauge fixings $\overrightarrow{\mathcal{K}} \approx 0$ will determine the internal three-center of mass $\vec{\eta}_{+}$in terms of the relative variables in an interaction-dependent way, and the Hamiltonian $H=M c$ depends on the interaction.

In the two-body model of [24] and in the N-body model of Section 4 of [35], the invariant mass has the form [93] $M c=\sum_{i}^{1, \ldots, N} E_{i}(\tau)$ with $E_{i}(\tau)=\sqrt{m_{i}^{2} c^{2}+\vec{\kappa}_{i}^{2}(\tau)+V_{i}(\tau)}$, where $V_{i}(\tau)=$ $\tilde{V}_{i}\left(\vec{\eta}_{m}(\tau)-\vec{\eta}_{n}(\tau), \vec{\kappa}_{k}\right)$ are suitable action-at-a-distance potentials. This implies $\epsilon p_{i}^{2}=m_{i}^{2} c^{2}+V_{i}(\tau)$. See Section 4 of [35] for the Poincare generators of an N-body system with action-at-a-distance interaction and Appendix A of [35] for the case of $\mathrm{N}$ charged particles plus Coulomb and Darwin potentials $[27,28]$.

In the till now existing relativistic kinetic theory and in relativistic statistical mechanics, $E_{i}=\sqrt{m_{i}^{2} c^{2}+\vec{\kappa}_{i}^{2}(\tau)}$ holds for a gas of non-interacting particles; no expression is known in the presence of interactions. Moreover, in all of the existing attempts to develop a relativistic kinetic theory, one always works by using the Cartesian four-coordinates $x^{\mu}$ of an arbitrary inertial frame centered on an arbitrary inertial time-like observer, so that the world-lines and the standard momenta of the particles have the expression $\tilde{x}_{i}^{\mu}\left(x^{o}\right)=\left(x^{o} ; \overrightarrow{\tilde{x}}_{i}\left(x^{o}\right)\right), \tilde{p}_{i \mu}\left(x^{o}\right)=\left(\tilde{p}_{i o}\left(x^{o}\right) ; \overrightarrow{\tilde{p}}_{i}\left(x^{o}\right)\right)$.

The absence till now of a satisfactory relativistic kinetic theory is due to the fact that the translation of the results found in the inertial rest frame in an arbitrary inertial frame is very complicated as shown in Section IIC of [35]. This is due to the fact that each particle has a different radar proper time, $x^{o}=x_{i}^{o}\left(\tau_{i}\right)$ when described in the three-spaces $\Sigma_{x^{0}=\text { const. }}$ and only on the solutions of Hamilton 
equations is it possible to find $\tau_{i}=\tau_{i}\left(x^{o}\right)$ and to study the complicated transition from the $6 \mathrm{~N}+1$ variables $\tau, \vec{z}, \vec{h}, \vec{\rho}_{a}(\tau), \vec{\pi}_{a}(\tau)$ associated with the rest frame Wigner three-space $\Sigma_{\tau}$ to the $6 \mathrm{~N}+1$ variables $x^{o}, \overrightarrow{\tilde{x}}_{i}\left(x^{o}\right), \overrightarrow{\tilde{p}}_{i}\left(x^{o}\right)$, associated with the Euclidean three-space $\Sigma_{x^{o}}$. The origin of all of these complications is connected to the absence of a unique notion of the relativistic center of mass like in non-relativistic physics.

\subsection{The Non-Relativistic Limit of the Inertial Rest Frame}

It is shown in $[31,34]$ that the description of N non-relativistic particles in Galilei space-time, with Newton positions $\vec{x}_{(n) i}(t)$ and Newton momenta $\vec{p}_{(n) i}(t)$, done in terms of the Newton center of mass $\vec{x}_{(n)}(t)$, with conjugate momentum $\vec{p}_{(n)}$, and of non-relativistic relative variables $\vec{\rho}_{(n) a}(t), \vec{\pi}_{(n)}(t)$, $a=1, \ldots, N-1$, can be obtained as the non-relativistic limit of the $3+1$ approach.

The limit of the inertial rest frame is the non-relativistic rest frame centered on the Newton center of mass and defined with the conditions $\vec{x}_{(n)}(t) \approx 0$ and $\vec{p}_{(n)} \approx 0$.

In it, the spatial part of the world-lines (2) becomes $\vec{x}_{i}(\tau) \rightarrow_{c \rightarrow \infty} \vec{x}_{(n) i}(t)=\vec{x}_{(n)}(t)+\overrightarrow{\tilde{\eta}}_{(n) i}(t) \approx \overrightarrow{\tilde{\eta}}_{(n) i}(t)$ with $\overrightarrow{\tilde{\eta}}_{(n) i}(t)=\vec{\eta}_{i}(\tau)$, because the non-relativistic limit of the gauge fixings eliminating the internal center of mass implies $\overrightarrow{\mathcal{K}} / c \approx 0 \rightarrow_{c \rightarrow \infty}-\sum_{i} m_{i} \overrightarrow{\tilde{\eta}}_{i}(t) \approx 0$. The particle momenta $\vec{\kappa}_{i}(\tau)=\overrightarrow{\tilde{\kappa}}_{i}(t)$ coincide with the non-relativistic momenta $\vec{p}_{(n) i}(t)$, because they collapse into momenta $\vec{\kappa}_{(n) i}(t)$ restricted by the rest frame condition $\overrightarrow{\mathcal{P}} \rightarrow_{c \rightarrow \infty} \sum_{i} \overrightarrow{\mathcal{K}}_{(n) i}(t) \approx 0$.

This is due to the fact that after the position $\tau=c t$, one can show that all of the time components of the three relativistic centers of mass $\left(\tilde{x}^{o} / c, Y^{o} / c, R^{o} / c\right)$ and of the individual particles $\left(x_{i}^{o} / c\right)$ tend to the absolute Newton time $t$ for $c \rightarrow \infty$. Moreover, for the spatial components, one has: $\overrightarrow{\tilde{x}}(\tau), \vec{Y}(\tau), \vec{R}(\tau) \rightarrow_{c \rightarrow \infty} \vec{x}_{(n)}(t), \vec{x}_{N W}(0)=\vec{z} / M c \rightarrow_{c \rightarrow \infty} \vec{x}_{(n)}(0)$ and $\vec{\eta}_{+}(\tau) \mapsto \vec{x}_{(n)}(t):$ the external and internal centers of mass and all of the relativistic collective variables collapse into the Newton center of mass. For the total momentum, we have $\vec{P}=\vec{p}_{(n)}$. Equation (2) imply $h^{\mu} \rightarrow_{c \rightarrow \infty}(1 ; 0)$, $\epsilon_{r}^{\mu}(\vec{h}) \rightarrow_{c \rightarrow \infty}\left(0 ; \delta_{r}^{i}\right)$ and $\vec{z}_{W}(\tau, \vec{\sigma}) \rightarrow_{c \rightarrow \infty} \vec{x}_{(n)}(t)+\vec{\sigma}$.

In the free case, the non-relativistic limit of the internal Poincaré generator $M c$ is $M c \rightarrow_{c \rightarrow \infty} m c+\mathcal{E}$ with $m=\sum_{i} m_{i}$ and $\mathcal{E}=\sum_{i} \frac{\vec{\kappa}_{(n) i}^{2}(t)}{2 m_{i}}$. For $\vec{S}$, one has $\vec{S} \rightarrow_{c \rightarrow \infty} \sum_{i} \vec{\eta}_{(n) i}(t) \times \vec{\kappa}_{(n) i}(t)=\vec{S}_{(n)}$. In the interacting case, a potential $V\left(\vec{\eta}_{(n) i}(t)-\vec{\eta}_{(n) j}(t), \vec{\kappa}_{(n) i}(t)\right)$ will be present in the relative Hamiltonian, i.e., in the internal energy $\mathcal{E}$.

As a consequence, in the non-relativistic rest frame $\vec{p}_{(n)} \approx 0, \vec{x}_{(n)} \approx 0$, there is an unfaithful internal Galilei algebra: $E_{\text {Galilei }}=H_{r e l}, \sum_{i} \vec{\kappa}_{(n) i} \approx 0, \vec{S}_{(n)}, \vec{K}_{(n)}=-\sum_{i} m_{i} \vec{\eta}_{(n) i}(t)-m \vec{x}_{(n)} \approx 0$.

In the free case, the non-relativistic limit of the external Poincaré generators $P^{\mu}, J^{i j}, K^{i}=J^{0 i}$, gives rise to the generators of the (external) Galilei algebra (centrally extended with the total mass $m$ ):

$$
\begin{array}{lll}
P^{o} & \rightarrow_{c \rightarrow \infty} & m c+E_{\text {Galilei, }} \quad E_{\text {Galilei }}=\frac{\vec{p}_{(n)}^{2}}{2 m}+\sum_{i} \frac{\vec{\kappa}_{(n) i}^{2}(t)}{2 m_{i}}=\sum_{i} \frac{\vec{p}_{(n) i}^{2}(t)}{2 m_{i}}, \\
\vec{P} & =\quad \vec{p}_{(n)}=\vec{P}_{\text {Galilei, }} \\
\vec{J} & =\quad \vec{x}_{(n)}(t) \times \vec{p}_{(n)}+\vec{S}_{(n)}=\sum_{i} \vec{x}_{(n) i}(t) \times \vec{p}_{(n) i}(t)=\vec{J}_{\text {Galilei },} \\
\frac{1}{c} \vec{K} \rightarrow_{c \rightarrow \infty} & t \vec{p}_{(n)}-m \vec{x}_{(n)}=\vec{K}_{\text {Galilei } .}
\end{array}
$$

Therefore, the non-relativistic limit of the relativistic N-body problem reproduces the Hamilton-Jacobi version of the non-relativistic N-body problem [31], which can be obtained with a canonical transformation implying $H_{\text {com }}=\frac{\vec{p}_{(n)}^{2}}{2 m} \mapsto H_{\text {com }}^{(H J)}=0$ for the Hamiltonian description of the free center of mass, so that the Hamiltonian is reduced to the internal energy $\mathcal{E}$.

\section{Extension to Non-Inertial Global Frames}

The description of an isolated system in an arbitrary relativistic non-inertial frame of Minkowski space-time with embedding $z^{\mu}\left(\tau, \sigma^{r}\right)=x^{\mu}(\tau)+F^{\mu}\left(\tau, \sigma^{r}\right)$, centered on the world-line $x^{\mu}(\tau)$ of an arbitrary time-like observer, of the $3+1$ approach is very complicated and still to be developed in 
detail, as shown in Section 3 of [35] and in [19,25,26,32,82]. The only relevant cases completely under control are pure differential rotations in Euclidean three-spaces without the pathologies of the rotating disk (see Equation (2.14) of [25]).

The only well-studied family of non-inertial frames comprises the relativistic non-inertial rest frames of an isolated system. They are all of the admissible $3+1$ splittings of Minkowski space-time whose instantaneous three-spaces $\Sigma_{\tau}$ tend to space-like hyper-planes orthogonal to the conserved four-momentum of the isolated system at spatial infinity, namely they tend to the Wigner three-spaces of the inertial rest frame asymptotically.

Their relevance is due to the fact that they are the only global non-inertial frames allowed by the equivalence principle (forbidding the existence of global inertial frames) in the canonical metric and tetrad Einstein gravity with matter [79-81], in globally hyperbolic, asymptotically flat (asymptotically Minkowskian) space-times without super-translations, so as to have the asymptotic ADM Poincaré group becoming the standard Poincare group of the matter in Minkowski space-time in the limit of the zero Newton constant.

These non-inertial frames can be centered on the external Fokker-Pryce center of inertia like the inertial ones and are described by the following embeddings (these embeddings are admissible if the functions $g(\tau, \vec{\sigma})$ and $g^{r}(\tau, \vec{\sigma})$ satisfy the restrictions on the four-metric ${ }^{4} g_{A B}\left(\tau, \sigma^{r}\right)=z_{A}^{\mu}\left(\tau, \sigma^{r}\right) \eta_{\mu \nu} z_{B}^{v}\left(\tau, \sigma^{r}\right)$ quoted at the beginning of Section 2 and in [87]):

$$
\begin{aligned}
& z^{\mu}(\tau, \vec{\sigma}) \approx z_{F}^{\mu}(\tau, \vec{\sigma})=Y^{\mu}(\tau)+u^{\mu}(\vec{h}) g(\tau, \vec{\sigma})+\epsilon_{r}^{\mu}(\vec{h})\left[\sigma^{r}+g^{r}(\tau, \vec{\sigma})\right], \\
& \rightarrow|\vec{\sigma}| \rightarrow \infty z_{W}^{\mu}(\tau, \vec{\sigma})=Y^{\mu}(\tau)+\epsilon_{r}^{\mu}(\vec{h}) \sigma^{r}, \quad x^{\mu}(\tau)=z_{F}^{\mu}(\tau, 0), \\
& g(\tau, 0)=g^{r}(\tau, 0)=0, \quad g(\tau, \vec{\sigma}) \rightarrow|\vec{\sigma}| \rightarrow \infty 0, \quad g^{r}(\tau, \vec{\sigma}) \rightarrow|\vec{\sigma}| \rightarrow \infty 0 .
\end{aligned}
$$

As shown in [26,27], the internal mass, the spin and the rest frame conditions and their gauge-fixings become [94]:

$$
\begin{aligned}
M c & =\int d^{3} \sigma\left(\frac{\operatorname{det}\left(\delta_{r}^{s}+\partial_{r} g^{s}\right)}{\sqrt{\gamma}} T_{F \perp \perp}-\partial_{r} g h_{F}^{r s} T_{F \perp s}\right)(\tau, \vec{\sigma}), \\
\hat{\mathcal{P}}^{u} & =\int d^{3} \sigma\left(-\frac{\delta^{u a} \epsilon_{a s r} \epsilon_{v w t} \partial_{v} g \partial_{w} g^{s} \partial_{t} g^{r}}{\sqrt{\gamma_{F}}} T_{F \perp \perp}\right. \\
& \left.-\left(\delta_{r}^{u}+\partial_{r} g^{u}\right) h_{F}^{r s} T_{F \perp s}\right)(\tau, \vec{\sigma}) \approx 0 . \\
\hat{\mathcal{K}}^{u} & =\int d^{3} \sigma\left(g\left[\delta^{u r} \partial_{r} g T_{F \perp \perp}-\left(\delta_{r}^{u}+\partial_{r} g^{u}\right) h_{F}^{r s} T_{F \perp s}\right]\right. \\
& \left.-\left(\sigma^{u}+g^{u}\right)\left[\frac{\operatorname{det}\left(\delta_{r}^{s}+\partial_{r} g^{s}\right)}{\sqrt{\gamma}} T_{F \perp \perp}-\partial_{r} g h_{F}^{r s} T_{F \perp s}\right]\right)(\tau, \vec{\sigma}) \approx 0, \\
\tilde{S}^{r} & \approx \hat{S}^{r}=\frac{1}{2} \delta^{r n} \epsilon_{n u v} \int d^{3} \sigma\left(\left(\sigma^{u}+g^{u}\right)\left[\delta^{v m} \partial_{m} g T_{F \perp \perp}-\left(\delta_{r}^{v}+\partial_{r} g^{v}\right) h_{F}^{r s} T_{F \perp s}\right]\right. \\
& \left.-\left(\sigma^{v}+g^{v}\right)\left[\delta^{u m} \partial_{m} g T_{F \perp \perp}-\left(\delta_{r}^{u}+\partial_{r} g^{u}\right) h_{F}^{r s} T_{F \perp s}\right]\right)(\tau, \vec{\sigma}) .
\end{aligned}
$$

Therefore, the non-inertial rest frame instant form of dynamics is well defined since we have: (a) a decoupled center of mass carrying a pole-dipole structure; (b) well-defined internal Poincaré generators $M c, \overrightarrow{\mathcal{P}} \approx 0, \overrightarrow{\tilde{S}}, \overrightarrow{\mathcal{K}} \approx 0$ at spatial infinity; (c) non-Euclidean three-spaces tending in a direction-independent way to space-like hyper-planes, where they are orthogonal to $P^{\mu}$. In $[26,27]$ is the determination of the effective Hamiltonian $\mathcal{M} c$ of the non-inertial rest frame instant form, which is not the internal mass $M c$, since $M c$ describes the evolution from the point of view of the asymptotic inertial observers. There is an additional term interpretable as an inertial potential producing relativistic inertial effects, and the final form of the effective Hamiltonian is $\mathcal{M} c=\int d^{3} \sigma \sqrt{\gamma\left(\tau, \sigma^{u}\right)}\left(\left(1+n_{F}\right) T_{F \perp \perp}+\right.$ $\left.n_{F}^{r} T_{F \perp r}\right)(\tau, \vec{\sigma})$.

\subsection{The Non-Relativistic Limit of the Non-Inertial Rest Frames}

The parametrized Galilei theories for isolated particle systems defined in [33] are the non-relativistic version of parametrized Minkowski theories: they imply a description of non-relativistic global 
non-inertial frames centered on accelerated observers, which is the limit of the relativistic $3+1$ splitting when there is the absolute Newton time $t$ and Euclidean three-spaces $\Sigma_{t}$. The Cartesian three-coordinates $x^{a}, a=1,2,3$, of an inertial frame in Galilei space-time centered on an inertial observer are expressed in terms of curvilinear three-coordinates $\sigma^{r}$, of a non-inertial frame centered on an accelerated observer $\vec{x}_{0}(t)=\overrightarrow{\mathcal{A}}(t, 0)$, by means of an invertible, global coordinate transformation $x^{a}=\mathcal{A}^{a}(t, \vec{\sigma}) \rightarrow|\vec{\sigma}| \rightarrow \infty A^{a}{ }_{b}(t) \sigma^{b}$, with inverse $\sigma^{r}=\mathcal{S}^{r}(t, \vec{x})$. The Jacobian of this transformation and its inverse are $J^{a}{ }_{r}(t, \vec{\sigma})=\frac{\partial \mathcal{A}^{a}(t, \vec{\sigma})}{\partial \sigma^{r}}(\operatorname{det} J(t, \vec{\sigma})>0), \tilde{J}^{r}{ }_{a}(t, \vec{\sigma})=\left[\frac{\partial \mathcal{S}^{r}(t, \vec{x}}{\partial x^{a}}\right]_{\vec{x}=\overrightarrow{\mathcal{S}}(t, \vec{\sigma})}\left(J^{a}{ }_{r}(t, \vec{\sigma}) \tilde{J}^{r}{ }_{b}(t, \vec{\sigma})=\delta_{b^{\prime}}^{a}\right.$ $\left.\tilde{J}_{a}^{s}(t, \vec{\sigma}) J^{a}{ }_{r}(t, \vec{\sigma})=\delta_{r}^{s}\right)$.

The non-relativistic action of [33], depending on the matter and on the functions $\overrightarrow{\mathcal{A}}(t, \vec{\sigma})$, is invariant under three-diffeomorphisms, so that the momenta $\vec{\rho}(t, \vec{\sigma})$, conjugate to the variables $\overrightarrow{\mathcal{A}}(t, \vec{\sigma})$, are determined by three first-class constraints: the variables $\overrightarrow{\mathcal{A}}(t, \vec{\sigma})$ are gauge variables, and a change of frame is a gauge transformation like in the relativistic case. In [34] is the expression of the ten generators of the Galilei algebra and of the effective non-inertial Hamiltonian $\left(\mathcal{M}_{\text {Galilei }}=E_{\text {Galilei }}-\left.\sum_{i} J^{r}{ }_{a}\left(t, \overrightarrow{\tilde{\eta}}_{i}(t)\right) \frac{\partial \mathcal{A}^{a}\left(t, \vec{\eta}_{i}(t)\right)}{\partial t}\right|_{\vec{\eta}_{i}(t)} p_{i r}(t)\right)$ in this framework. In the case of non-relativistic particles, one recovers the standard Euler, Jacobi, Coriolis and centrifugal forces in their equation of motion in the non-inertial frames described by the functions $\mathcal{A}^{a}(t, \vec{\sigma})=x_{o}^{a}(t)+\sigma^{r} R_{r a}(t)$ (see Equation (4.9) of [33]).

The non-relativistic limit of the embedding (6) of the relativistic non-inertial rest frame is done by putting $\vec{h}=\frac{\vec{v}}{c}+O\left(c^{-2}\right)$ and $\sigma^{r}+g^{r}(\tau, \vec{\sigma}) \rightarrow_{c \rightarrow \infty} \mathcal{A}^{r}(t, \vec{\sigma})$ and by assuming $g(\tau, \vec{\sigma})=O\left(c^{-2}\right)$. Then, we get $\frac{1}{c} z^{o}(\tau, \vec{\sigma}) \rightarrow_{c \rightarrow \infty} t, \vec{z}(\tau, \vec{\sigma}) \rightarrow_{c \rightarrow \infty} \vec{y}_{o}+\vec{v} t+\overrightarrow{\mathcal{A}}(t, \vec{\sigma})$.

If we put $\vec{x}_{o}(t)=\overrightarrow{\mathcal{A}}(t, \overrightarrow{0})=0$ and $\vec{p}_{(n)}=0$, we see that we are in a Newtonian non-inertial rest frame centered on the Newton center of mass $\vec{x}_{(n)}(t)=\vec{y}_{o}+\vec{v} t$.

The previous conditions imply that the Jacobian is $J^{a}{ }_{r}=\delta_{r}^{a}+\frac{\partial g^{a}}{\partial \sigma^{r}}$ (with inverse $\tilde{J}^{r}{ }_{a}$ ), and the induced three-metric on the Euclidean three-space is $h_{r s}(\tau, \vec{\sigma})=-\epsilon g_{r s}(\tau, \vec{\sigma})=H_{r s}(t, \vec{\sigma})+O\left(c^{-2}\right)$ with $H_{r s}=\sum_{a} J^{a}{ }_{r} J^{a}{ }_{s}=\delta_{r s}+\frac{\partial g^{r}}{\partial \sigma^{s}}+\frac{\partial g^{s}}{\partial \sigma^{r}}+\sum_{u} \frac{\partial g^{u}}{\partial \sigma^{r}} \frac{\partial g^{u}}{\partial \sigma^{s}}$ (its inverse is $H^{r s}=\sum_{a} \tilde{J}^{r} \tilde{J}_{a}^{s}$ ). With these notations, the non-relativistic limit of the external and internal Poincaré generators leads to the following form for the Galilei generators:

$$
\begin{aligned}
P^{o} & \rightarrow_{c \rightarrow \infty} \quad m c+E_{\text {Galilei, }} \quad E_{\text {Galilei }}=H_{c}=\frac{\vec{p}_{(n)}^{2}}{2 m}+\mathcal{E}_{\text {Galilei, }} \\
\vec{P} & =\quad \vec{p}_{(n)}=\vec{P}_{\text {Galilei, }} \\
\vec{J} & =\quad \vec{x}_{(n)}(t) \times \vec{p}_{(n)}+\overrightarrow{\mathcal{S}}_{\text {Galilei }}=\vec{J}_{\text {Galilei, }} \\
\frac{1}{c} \vec{K} & \rightarrow_{c \rightarrow \infty} \quad t \vec{p}_{(n)}-m \vec{x}_{(n)}=\vec{\kappa}_{\text {Galilei, }} \\
\mathcal{E}_{\text {Galilei }} & =\sum_{i} \frac{1}{2 m_{i}} H^{r s}\left(t, \overrightarrow{\tilde{\eta}}_{i}(t)\right) \tilde{\kappa}_{i r}(t) \tilde{\kappa}_{i s}(t), \\
\mathcal{S}_{\text {Galilei }}^{r} & =\sum_{u v} \epsilon^{r u v} \sum_{i} \mathcal{A}^{u}\left(t, \overrightarrow{\tilde{\eta}}_{i}(t)\right) J^{v} r\left(t, \overrightarrow{\tilde{\eta}}_{i}(t)\right) \quad \sum_{s} H^{r s}\left(t, \overrightarrow{\tilde{\eta}}_{i}(t)\right) \tilde{\kappa}_{i s}(t), \\
\mathcal{P}_{\text {Galilei }}^{r} & =\sum_{\text {ius }} J^{r}\left(t, \overrightarrow{\tilde{\eta}}_{i}(t)\right) H^{r s}\left(t, \overrightarrow{\tilde{\eta}}_{i}(t)\right) \tilde{\kappa}_{i s}(t) \approx 0, \\
\mathcal{K}_{\text {Galilei }}^{r}= & -\sum_{i} m_{i} \mathcal{A}^{r}\left(t, \overrightarrow{\tilde{\eta}}_{i}(t)\right) \approx 0 .
\end{aligned}
$$

\section{The Relativistic Micro-Canonical Ensemble in Inertial Rest Frames}

The either non-relativistic or relativistic isolated system of $N$ scalar particles with arbitrary either short- or long-range interactions described in inertial and non-inertial frames in the previous sections will be used for the definition of the micro-canonical ensemble in both in inertial and non-inertial rest frames.

In non-relativistic inertial frames, the micro-canonical distribution function $f_{(m c, n r, s t)}\left(\vec{x}_{1}, . ., \vec{p}_{N} \mid E, V, N\right)$ and the micro-canonical partition function $Z_{(n r, s t)}(E, V, N)$ of such an isolated system with Newtonian Hamiltonian $H_{N}$ depend on the volume $V$ [95], on the particle number $N$ and on the value $E=H_{N}$ of the total conserved energy (see for instance Chapter 6 of [1]) 
and have the following expression (the indices have the following meaning: " $\mathrm{mc}$ " for micro-canonical, "nr" for non-relativistic, "st" for statistical):

$$
\begin{gathered}
f_{(m c, n r, s t)}\left(\vec{x}_{1}, . ., \vec{p}_{N} \mid E, V, N\right)=\frac{1}{Z_{(n r, s t)}(E, V, N)} \chi(V) \delta\left(H_{N}\left(\vec{x}_{1}, . ., \vec{p}_{N}\right)-E\right), \\
\chi(V)=1 \text { for } \vec{x}_{i} \in V, \quad \chi(V)=0 \quad \text { for } \vec{x}_{i} \notin V, \\
Z_{(n r, s t)}(E, V, N)=\int d \Gamma_{N} \chi(V) \delta\left(H_{N}\left(\vec{x}_{1}, . ., \vec{p}_{N}\right)-E\right)=\frac{\partial \Omega_{(n r, s t)}(E, V, N)}{\partial E}, \\
\Omega_{(n r, s t)}(E, V, N)=\int d \Gamma_{N} \chi(v) \theta\left(H\left(\vec{x}_{1}, . ., \vec{p}_{N}\right)-E\right), \\
d \Gamma_{N}=(N !)^{-1} \prod_{i=1, \ldots, N} d^{3} x_{i} d^{3} p_{i}, \\
\int d \Gamma_{n} f_{(m c, n r, s t)}\left(\vec{x}_{1}, . ., \vec{p}_{N} \mid E, V, N\right)=1 .
\end{gathered}
$$

Due to the Hamilton equations of the particles, the Liouville theorem implies $\frac{\partial f_{(m c, n, s t)}}{\partial t}+$ $\left\{f_{(m c, n r, s t)}, H\right\}=\hat{L} f_{(m c, n r, s t)}=0$, where $\hat{L}=\frac{\partial}{\partial t}+\sum_{i}\left(\frac{\partial H}{\partial \vec{p}_{i}} \cdot \frac{\partial}{\partial \vec{x}_{i}}-\frac{\partial H}{\partial \vec{x}_{i}} \cdot \frac{\partial}{\partial \vec{p}_{i}}\right)$ is the Liouville operator.

The isolation of the system implies $\frac{\partial f_{(m c, n, s t)}}{\partial t}=0$, namely equilibrium statistical mechanics.

For $\mathrm{N}$ free particles of mass $m$ in a spherical volume $V=\frac{4}{3} \pi R^{3}\left(\chi(V)=0\right.$ for $\vec{x}_{i}^{2}>R^{2}$ and $\chi(V)=1$ for $\vec{x}_{i}^{2}<R^{2}$ ) with canonical coordinates $\vec{x}_{i}, \vec{p}_{i}, i=1, \ldots, N$, and Hamiltonian $H=\sum_{i=1}^{\infty} \frac{\vec{p}_{i}^{2}}{2 m}$, the micro-canonical distribution function has the expression (see Equation (C5) of Appendix $C$ of $[35,64]$ ):

$$
Z_{(n r, s t)}(E, V, N)=\frac{1}{N !} \frac{(\sqrt{2 \pi m})^{3 N} E^{3 N / 2} V^{N}}{E \Gamma(3 N / 2)} \theta(E) .
$$

In the micro-canonical ensemble, the statistical average of a function $F\left(\vec{x}, \vec{p} ; \vec{x}_{i}, \vec{p}_{i}\right)$ is:

$$
F_{(m c)}(\vec{x}, \vec{p} \mid E, V, N)=<F>_{(m c)}=\int d \Gamma_{N} F\left(\vec{x}, \vec{p} ; \vec{x}_{i}, \vec{p}_{i}\right) f_{(m c, n r, s t)}\left(\vec{x}_{1}, \ldots, \vec{p}_{N} \mid E, V, N\right) .
$$

The non-relativistic extended micro-canonical distribution and partition functions used in [43] for the case of a Hamiltonian with long-range Newtonian gravity interactions [96] depend not only on the volume $V$, on the particle number $N$ and on the value $E$ of the total conserved energy $(H=E)$, but also on the value $\overrightarrow{\mathcal{S}}$ of the total conserved angular momentum:

$$
\begin{aligned}
\tilde{f}_{(m c, n r, s t)}\left(\vec{x}_{1}, \ldots, \vec{p}_{N} \mid E, \overrightarrow{\mathcal{S}}, V, N\right)= & \tilde{Z}_{(n r, s t)}^{-1}(E, \overrightarrow{\mathcal{S}}, V, N) \chi(V) \delta\left(H_{N}\left(\vec{x}_{1}, . ., \vec{p}_{N}\right)-E\right) \delta^{3}\left(\vec{S}_{N}-\overrightarrow{\mathcal{S}}\right), \\
\tilde{Z}_{(n r, s t)}(E, \overrightarrow{\mathcal{S}}, V, N)= & \int d \Gamma_{N} \chi(V) \delta\left(H_{N}\left(\vec{x}_{1}, . ., \vec{p}_{N}\right)-E\right) \delta^{3}\left(\vec{S}_{N}-\overrightarrow{\mathcal{S}}\right), \\
& Z_{(n r, s t)}(E, V, N)=\int d^{3} \mathcal{S} \tilde{Z}_{(n r, s t)}(E, \overrightarrow{\mathcal{S}}, V, N) .
\end{aligned}
$$

These definitions can be extended to the non-relativistic inertial rest frame of Section 2.2 by putting the center of mass of the isolated system at the origin of the three-coordinates $\left(\vec{x}_{(n)}=0\right)$ and by replacing the total energy $E=\frac{\vec{p}_{(n)}^{2}}{2 N m}+\mathcal{E}$ with the internal energy $\mathcal{E}$, which is an invariant of the centrally extended Galilei algebra, due to the rest frame condition $\vec{p}_{(n)} \approx 0$. The ordinary and extended micro-canonical partition functions in the inertial rest frame $\tilde{Z}_{(n r)}(\mathcal{E}, V, N)$ and $\tilde{Z}_{(n r)}(\mathcal{E}, \overrightarrow{\mathcal{S}}, V, N)$ will be defined in Section 4.1 by using only the internal Galilei generators of the isolated system. In the non-relativistic setting, it is possible to reintroduce the dependence on the center of mass and to recover the original partition functions (8) and (12).

In Section 4.2 is the definition of the relativistic ordinary and extended micro-canonical partition functions, $\tilde{Z}(\mathcal{E}, V, N)$ and $\tilde{Z}(\mathcal{E}, \overrightarrow{\mathcal{S}}, V, N)$, in the relativistic rest frame of Section 2.1. Now, $\mathcal{E}$ is the conserved invariant mass $M c$ of the isolated system $(\overrightarrow{\mathcal{S}}$ is its rest spin), and it is not possible to reintroduce a dependence on the external center of mass as in the non-relativistic case due to the non-covariance of the Jacobi data $\vec{z}$. 


\subsection{The Micro-Canonical Ensemble in the Non-Relativistic Inertial Rest Frame}

In the non-relativistic inertial rest frame of the N-particle system defined in Section 2.2, one has $\vec{x}_{(n)}=\vec{p}_{(n)}=0$. The Newtonian center of mass is at rest and is chosen as the origin of the three-coordinates of the Euclidean three-spaces. The volume $V$ is assumed to have the center in the origin. The generators of the Galilei group are given in Equation (4) and are function of relative variables $\vec{\rho}_{(n) a}$ and $\vec{\pi}_{(n) a}, a=1, \ldots, N-1$.

From Section 2.2 and Equation (4), we get the following expression for the extended and ordinary micro-canonical partition functions for an isolated system of $\mathrm{N}$ particles:

$$
\begin{aligned}
& \tilde{Z}_{(n r)}(\mathcal{E}, \overrightarrow{\mathcal{S}}, V, N)=\frac{1}{N !} \int \prod_{i}^{1 . . N} d^{3} \eta_{i} \chi(V) \int \prod_{j}^{1 . . N} d^{3} \kappa_{j} \delta\left(E_{\text {Galilei, }, N}-\mathcal{E}\right) \\
& \delta^{3}\left(\vec{S}_{\text {Galilei, }, N}-\overrightarrow{\mathcal{S}}\right) \delta^{3}\left(\overrightarrow{\mathcal{P}}_{\text {Galilei }, N}\right) \delta^{3}\left(\frac{\overrightarrow{\mathcal{K}}_{\text {Galilei,N }}}{m}\right) \\
& =\frac{1}{N !} \int \prod_{a}^{1 \ldots N-1} d^{3} \rho_{(n) a} \chi(V) \int \prod_{b}^{1 \ldots N-1} d^{3} \pi_{(n) b} \\
& \delta\left(E_{\text {Galilei }, N}-\mathcal{E}\right) \delta^{3}\left(\vec{S}_{\text {Galilei, } N}-\overrightarrow{\mathcal{S}}\right), \\
& \tilde{Z}_{(n r)}(\mathcal{E}, V, N)=\int d^{3} \mathcal{S} \tilde{Z}_{(n r)}(\mathcal{E}, \overrightarrow{\mathcal{S}}, V, N) \\
& =\frac{1}{N !} \int \prod_{i}^{1 . . N} d^{3} \eta_{i} \chi(V) \int \prod_{j}^{1 . N} d^{3} \kappa_{j} \delta\left(E_{\text {Galile }, N}-\mathcal{E}\right) \\
& \delta^{3}\left(\overrightarrow{\mathcal{P}}_{\text {Galilei,N }}\right) \delta^{3}\left(\frac{\overrightarrow{\mathcal{K}}_{\text {Galilei,N }}}{m}\right) \\
& =\frac{1}{N !} \int \prod_{a}^{1 . . N-1} d^{3} \rho_{(n) a} \chi(V) \int \prod_{b}^{1 . . N-1} d^{3} \pi_{(n) b} \delta\left(E_{\text {Galilei, },}-\mathcal{E}\right) .
\end{aligned}
$$

The ordinary micro-canonical distribution function $\tilde{Z}_{(n r)}(\mathcal{E}, V, N)$ is equal to the standard one with $N-1$ particles having as canonical coordinates a canonical basis of relative ones, $\vec{\rho}_{(n) a}, \vec{\pi}_{(n) a}, a=1, \ldots, N-1$, with the energy $E_{\text {Galilei, } N}=\frac{1}{2 m} \sum_{a}^{1 . N-1} \vec{\pi}_{(n) a}^{2}+$ potentials.

For the micro-canonical distribution function, we have:

$$
\begin{aligned}
\tilde{f}_{(m c, n r)} & \left.\left(\vec{\eta}_{1}, . ., \overrightarrow{\mathcal{K}}_{N} \mid \mathcal{E}, V, N\right)\right|_{\vec{x}_{(n)}=\vec{p}_{(n)}=0}=\tilde{Z}_{(n r)}^{-1}(\mathcal{E}, V, N) \frac{\chi(V)}{N !} \delta\left(E_{\text {Galilei, }}-\mathcal{E}\right) \\
& \delta^{3}\left(\vec{S}_{\text {Galilei, },}-\overrightarrow{\mathcal{S}}\right) \delta^{3}\left(\overrightarrow{\mathcal{P}}_{\text {Galilei }, N}\right) \delta^{3}\left(\frac{\overrightarrow{\mathcal{K}}_{\text {Galilei,N }}}{m}\right) \approx \tilde{f}_{(m c, n r)}\left(\vec{\rho}_{(n) 1}, . ., \vec{\pi}_{(n) N-1} \mid \mathcal{E}, V, N\right) .
\end{aligned}
$$

It satisfies the Liouville equation with the Hamilton-Jacobi Hamiltonian $H=\mathcal{E}=\left.E_{G a l i l e i}\right|_{\vec{P}_{(n)}=\vec{x}_{(n)}=0}$. Moreover, it satisfies $\partial_{t} f_{(m c, n r)}=0$, so that it is an equilibrium distribution function in statistical mechanics. The statistical average of a function $F\left(\vec{x}, \vec{p} ; \vec{x}_{(n) i}, \vec{p}_{(n) i}\right)$ is defined as in Equation (10).

In the non-relativistic case, by using the results in Section 2.2, we can undo the Hamilton-Jacobi transformation to the center of mass, and we can recover Equation (8) from the second of Equation (12) in a sphere of radius $\mathrm{R}$ :

$$
\tilde{Z}_{(n r, s t)}(E, V, N)=\int \prod_{i}^{1 \ldots N} d^{3} x_{(n) i} d^{3} p_{(n) i} \theta\left(R-\left|\vec{x}_{(n)}\right|\right) \tilde{Z}_{(n r)}\left(E=\frac{\vec{p}_{(n)}^{2}}{2 m}+\mathcal{E}, V, N\right) .
$$

This is possible because the Galilei energy generator is the sum of the kinetic energy of the center of mass and of the internal energy, which is invariant at the non-relativistic level. This property does not exist at the relativistic level with the Poincaré group.

As shown in Appendix C of [35] (Equations (C17), (C42) and (C43) with $\vec{\kappa}_{+}=0$ ), after a long calculation, one gets the following expressions for the standard and the extended micro-canonical distribution functions in the inertial rest frame for $\mathrm{N}$ free particles of mass $m\left(x^{2} j_{1}(x)=\sin x-x \cos x\right)$ : 


$$
\begin{gathered}
\tilde{Z}_{(n r)}(\mathcal{E}, V, N)=\frac{1}{N !}\left(\sqrt{\frac{m}{2 \pi}}\right)^{3 N} \frac{\mathcal{E}^{(3 N-5) / 2}}{\Gamma((3 N-3) / 2)} \theta(\mathcal{E}) \\
\quad \sqrt{\frac{32 \pi}{N^{3} m^{9}}} 3^{N-1} V^{N-1} \int_{0}^{\infty} x^{2} d x\left(\frac{j_{1}(x)}{x}\right)^{N}, \\
\tilde{Z}_{(n r)}(\mathcal{E}, V, N, \overrightarrow{\mathcal{S}})=\frac{1}{N !(2 \pi)^{9 N}}\left(\sqrt{8 \pi^{3}}\right)^{N+1}\left(\sqrt{m^{3}}\right)^{N-1} \frac{(2 \pi)^{3}}{(m)^{3}}\left(\frac{3 V}{4 \pi}\right)^{N-1} \\
\times \prod_{i}^{N} \int_{0}^{1} x_{i}^{2} d x_{i} \int_{0}^{\pi} \sin \theta_{i} d \theta_{i} \int_{0}^{2 \pi} d \phi_{i} \delta^{3}\left(\sum_{i=1}^{N} \vec{x}_{i}\right) \frac{\left(\mathcal{E}-\tilde{\mathcal{E}}\left(\vec{\eta}_{i}, \overrightarrow{\mathcal{S}}\right)\right)^{(3 N-3) / 2-1}}{\Gamma((3 N-3) / 2)} \theta\left(\mathcal{E}-\tilde{\mathcal{E}}\left(\vec{\eta}_{i}, \overrightarrow{\mathcal{S}}\right)\right),
\end{gathered}
$$

in which:

$$
\begin{aligned}
\tilde{\mathcal{E}}\left(\vec{\eta}_{i}, \overrightarrow{\mathcal{S}}\right) & =\frac{\left(\mathcal{S}^{1}\right)^{2}}{2 m \sum_{i=1}^{N}\left(\eta_{i}^{1}\right)^{2}}+\frac{\left(\mathcal{S}^{2}\right)^{2}}{2 m \sum_{i=1}^{N}\left(\eta_{i}^{2}\right)^{2}}+\frac{\left(\mathcal{S}^{3}\right)^{2}}{2 m \sum_{i=1}^{N}\left(\eta_{i}^{3}\right)^{2}} \\
& =\frac{1}{2 m R^{2}}\left(\frac{\left(\mathcal{S}^{1}\right)^{2}}{\sum_{i=1}^{N} x_{i}^{2} \cos ^{2} \phi_{i} \sin ^{2} \theta_{i}}+\frac{\left(\mathcal{S}^{2}\right)^{2}}{\sum_{i=1}^{N} x_{i}^{2} \sin ^{2} \phi_{i} \sin ^{2} \theta_{i}}+\frac{\left(\mathcal{S}^{3}\right)^{2}}{2 m \sum_{i=1}^{N} x_{i}^{2} \cos ^{2} \theta_{i}}\right) .
\end{aligned}
$$

\subsection{The Micro-Canonical Ensemble in the Relativistic Inertial Rest Frame}

Let us remark that in the relativistic case, there exists the following definition of the standard micro-canonical partition function [9] for $\mathrm{N}$ free particles of equal mass with world-lines $x_{i}^{\mu}$, conjugate momenta $p_{i}^{\mu}$ and Hamiltonian $H_{N}=\sum_{i}^{1 \ldots N} \sqrt{m^{2} c^{2}+\vec{p}_{i}^{2}}$ :

$$
Z_{(s t)}(E, V, N)=\frac{1}{N !} \int \chi(V) \prod_{i}^{1 . . N} d^{3} x_{i} d^{3} p_{i} \delta\left(H_{N}-E\right),
$$

in an arbitrary inertial frame of Minkowski space-time (see Endnote [97]—which refers to [98]). This definition is obtained by putting by hand $x_{1}^{o}=\ldots=x_{N}^{o}=x^{o}$ and by using the mass-shell conditions to eliminate the energies $p_{i}^{o ' s}$.

However, no one was able to introduce interactions in this approach.

As shown in Section 2, to get a consistent relativistic formulation in the presence of every type of interaction, one has to use the $3+1$ splitting method, to eliminate the non-covariant non-local (therefore, non-measurable) four-center of mass (described by the Jacobi data $\vec{z}, \vec{h}$ ) and to describe the particles with the Wigner three-vectors $\vec{\eta}_{i}(\tau)$ and $\vec{\kappa}_{i}(\tau), N=1, \ldots, N$, as fundamental canonical variables together with the rest frame conditions eliminating the internal three-center of mass inside the instantaneous Wigner three-spaces of the inertial rest frame (centered on the Fokker-Pryce external four-center of inertia with Jacobi three-velocity $\vec{h}=0$ ) and reformulating the dynamics in terms of relative variables $\vec{\rho}_{a}$ and $\vec{\pi}_{a}, a=1, \ldots, N-1$.

The relativistic micro-canonical partition function is defined in terms of the internal Poincare generators living inside the Wigner three-spaces $\Sigma_{\tau}$ of the inertial rest frame. It is a function of the internal energy $\mathcal{E}$ [99], of the rest spin $\overrightarrow{\mathcal{S}}$ in the rest frame and of the volume V [100].

The extended and ordinary partition functions of the micro-canonical ensemble are:

$$
\begin{aligned}
\tilde{Z}(\mathcal{E}, \overrightarrow{\mathcal{S}}, V, N)= & \frac{1}{N !} \int \prod_{i}^{1 . . N} d^{3} \eta_{i} \chi(V) \int \prod_{j}^{1 . . N} d^{3} \kappa_{j} \delta\left(M_{N} c-\mathcal{E}\right) \\
& \delta^{3}\left(\vec{S}_{N}-\overrightarrow{\mathcal{S}}\right) \delta^{3}\left(\overrightarrow{\mathcal{P}}_{N}\right) \delta^{3}\left(\frac{\overrightarrow{\mathcal{K}}_{N}}{M_{N} c}\right) \\
= & \frac{1}{N !} \int d^{3} \eta \prod_{a=1}^{N-1} d^{3} \rho_{a} \chi(V) \int \prod_{b}^{1 . . N-1} d^{3} \pi_{b} J\left(\vec{\rho}_{a}, \vec{\pi}_{a}\right) \delta^{3}\left(\vec{\eta}-\vec{\eta}_{+}\left(\vec{\rho}_{a}, \vec{\pi}_{a}\right)\right) \\
& \delta\left(\hat{M}_{N}\left(\vec{\rho}_{a}, \vec{\pi}_{a}\right) c-\mathcal{E}\right) \delta^{3}\left(\sum_{a=1}^{N-1} \vec{\rho}_{a} \times \vec{\pi}_{a}-\overrightarrow{\mathcal{S}}\right), \\
\tilde{Z}(\mathcal{E}, V, N)= & \int d^{3} \mathcal{S} \tilde{Z}(\mathcal{E}, \overrightarrow{\mathcal{S}}, V, N) \\
= & \frac{1}{N !} \int \prod_{i}^{1 . N} d^{3} \eta_{i} \chi(V) \int \prod_{j}^{1 . . N} d^{3} \kappa_{j} \delta\left(M_{N} c-\mathcal{E}\right) \delta^{3}\left(\overrightarrow{\mathcal{P}}_{N}\right) \delta^{3}\left(\overrightarrow{\mathcal{K}}_{N}\right) \\
= & \frac{1}{M_{N} c} \int d^{3} \eta \prod_{a=1}^{N-1} d^{3} \rho_{a} \chi(V) \int \prod_{b}^{1 . . N-1} d^{3} \pi_{b} J\left(\vec{\rho}_{a}, \vec{\pi}_{a}\right) \delta^{3}\left(\vec{\eta}-\vec{\eta}_{+}\left(\vec{\rho}_{a}, \vec{\pi}_{a}\right)\right) \\
& \delta\left(\hat{M}_{N}\left(\vec{\rho}_{a}, \vec{\pi}_{a}\right) c-\mathcal{E}\right) .
\end{aligned}
$$

Their explicit form is not known either in the case of free particles. 
Since the three-vectors $\vec{\eta}_{i}(\tau), \vec{\kappa}_{i}(\tau), \vec{\rho}_{a}(\tau), \vec{\pi}_{a}(\tau)$ are Wigner spin-1 three-vectors, the invariant mass $M c$ is a Lorentz scalar. $\vec{S}, \overrightarrow{\mathcal{P}}$ and $\overrightarrow{\mathcal{K}} / M c$ are Wigner spin- 1 three-vectors: under a Lorentz transformation $\Lambda$, they undergo a Wigner rotation $R(\Lambda)$, so that expressions like $\delta^{3}(\overrightarrow{\mathcal{P}})$ are Lorentz scalars. Furthermore, the volume V, defined in [100] , is a Lorentz scalar because both $\left|\vec{\eta}_{i}(\tau)\right|$ and $\left|\vec{\rho}_{a}(\tau)\right|$ are Lorentz scalars. This is a byproduct of the use of Lorentz-scalar radar four-coordinates in this new formulation of relativistic mechanics with its special inertial frames. As said at the end of Section 2.1, in an ordinary inertial frame centered on an inertial observer A with Cartesian four-coordinates $x^{\mu}$, the world-lines of the particles would be $\tilde{x}_{i}^{\mu}\left(x^{o}\right)=\left(x^{o} ; \vec{x}_{i}\left(x^{o}\right)\right)$, and a volume $\tilde{V}$ would be defined as a spherical box centered on A in the three-spaces $\Sigma_{x^{0}=\text { const. }}|| \overrightarrow{\tilde{x}}_{i}\left(x^{0}\right) \mid<R$. The spherical box $\tilde{V}$ centered on $\mathrm{A}$ is the standard non-Lorentz-invariant type of volume. As said in Section 2.1, it is extremely complicated to make explicit the transition between the two formulations.

As a consequence, $\tilde{Z}(\mathcal{E}, V, N)$ is a Lorentz scalar, while one gets $\tilde{Z}(\mathcal{E}, \overrightarrow{\mathcal{S}}, V, N) \mapsto$ $\tilde{Z}\left(\mathcal{E}, R(\Lambda)^{-1} \overrightarrow{\mathcal{S}}, V, N\right)$ under a Lorentz transformation.

For the relativistic ordinary and extended distribution functions, one has:

$$
\begin{aligned}
f_{(m c)}\left(\vec{\rho}_{1}, . ., \vec{\pi}_{N-1} \mid \mathcal{E}, V, N\right)= & \tilde{Z}^{-1}(\mathcal{E}, V, N) \frac{\chi(V)}{N !} \delta\left(M_{N} c-\mathcal{E}\right) \delta^{3}\left(\overrightarrow{\mathcal{P}}_{N}\right) \delta^{3}\left(\frac{\overrightarrow{\mathcal{K}}_{N}}{M_{N} c}\right), \\
\tilde{f}_{(m c)}\left(\vec{\rho}_{1}, . ., \vec{\pi}_{N-1} \mid \mathcal{E}, \overrightarrow{\mathcal{S}}, V, N\right)= & \tilde{Z}^{-1}(\mathcal{E}, \overrightarrow{\mathcal{S}}, V, N) \frac{\chi(V)}{N !} \delta\left(M_{N} c-\mathcal{E}\right) \delta^{3}\left(\vec{S}_{N}-\overrightarrow{\mathcal{S}}\right) \\
& \delta^{3}\left(\overrightarrow{\mathcal{P}}_{N}\right) \delta^{3}\left(\frac{\overrightarrow{\mathcal{K}}_{N}}{M_{N} c}\right) .
\end{aligned}
$$

$f_{(m c)}$ satisfies the Liouville theorem with $H=M c$. Moreover, it is time independent, $\partial_{\tau} f_{(m c)}=0$, so that it is an equilibrium distribution function in relativistic statistical mechanics.

As said at the end of Section 2.1, a manifestly covariant micro-canonical distribution function of the type $F_{(m c)}\left(\overrightarrow{\tilde{x}}_{i}\left(x^{o}\right), \overrightarrow{\tilde{p}}_{i}\left(x^{o}\right) \mid \mathcal{E}, V, N\right)$, i.e., depending on the world-lines and their momenta in an arbitrary Lorentz frame (like in all of the existing approaches) does not exist due to the non-covariance of the Jacobi data $\vec{z}$ of the canonical external four-center of mass. In group-theoretical terms, the basic obstruction to get this type of distribution function is that the Poincare energy cannot be written as the center of mass energy plus an internal energy, like in the case of the Galilei group.

\section{The Micro-Canonical Ensemble in Non-Inertial Frames}

Furthermore, for the non-relativistic case, there is no definition of the micro-canonical ensemble of an isolated system of particles with either short- or long-range interactions in non-inertial frames, where all of the extra inertial forces are long range.

In this section is the definition of the two micro-canonical partition functions in the relativistic non-inertial rest frame of Section 3, and it is shown how to make the non-relativistic limit the non-relativistic non-inertial rest frame.

The definition of the non-rest non-inertial frames is possible, but extremely complicated, as shown in Section 3 and in [35]. In all of these cases, the system of particles are isolated inside the Lorentz scalar non-dynamical volume $\mathrm{V}$ determined only by the range of the forces. In going to non-inertial frames, only the variables defining the volume are passively changed with a mathematical transformation. The present formulation cannot be used for a gas in a box: in this case, the isolated system should be composed by the gas plus a dynamically described box.

\subsection{The Micro-Canonical Ensemble in the Relativistic Non-Inertial Rest Frames}

The definition (19) of the extended micro-canonical partition function can be used in the relativistic non-inertial rest frames by replacing the internal Poincaré generators with the 10 asymptotic Poincaré generators given in Equation (6) (see also $[25,26]$ ). The non-inertial Hamiltonian is the internal mass 
Mc. The non-inertial extended partition function, depending on the inertial potentials $g$ and $g^{r}$ appearing in Equation (6), is:

$$
\begin{aligned}
\tilde{Z}\left(\mathcal{E}, \overrightarrow{\mathcal{S}}, V, N \mid g, g^{r}\right)= & \frac{1}{N !} \int \chi(V) \prod_{i=1}^{N} d^{3} \eta_{i} d^{3} \kappa_{i} \delta\left(\mathcal{M}_{N}\left(\vec{\eta}_{i}, \vec{\kappa}_{i} ; g, g^{r}\right) c-\mathcal{E}\right) \\
& \delta^{3}\left(J_{N}^{u}\left(\vec{\eta}_{i}, \vec{\kappa}_{i} ; g, g^{r}\right)-\mathcal{S}^{u}\right) \delta^{3}\left(\hat{\mathcal{P}}_{N}^{u}\left(\vec{\eta}_{i}, \vec{\kappa}_{i} ; g, g^{r}\right)\right) \delta^{3}\left(\frac{\hat{\mathcal{K}}_{N}^{u}\left(\vec{\eta}_{i}, \vec{\kappa}_{i} ;, g, g^{r}\right)}{\mathcal{M}_{N}\left(\vec{\eta}_{i}, \dot{\kappa}_{i}, g, g^{r}\right) c}\right),
\end{aligned}
$$

In non-inertial relativistic frames, relative variables cannot be tensorially defined in their non-Euclidean three-spaces. In the interacting case with potentials $V\left(\vec{\eta}_{i}(\tau)-\vec{\eta}_{j}(\tau) ; \vec{\kappa}_{i}(\tau)\right)=\tilde{V}\left(\sqrt{\left(\vec{\eta}_{i}(\tau)-\vec{\eta}_{j}(\tau)\right)^{2}} ; \vec{k}_{i}(\tau)\right)$, one must replace the quantity $\left(\vec{\eta}_{i}(\tau)-\vec{\eta}_{j}(\tau)\right)^{2}$ with the bi-scalar Synge world function for Riemannian three-spaces $\sigma_{(i j)}\left(\vec{\eta}_{i}(\tau), \vec{\eta}_{j}(\tau)\right)=\frac{1}{2}\left(\lambda_{1}-\lambda_{0}\right) \int_{\lambda_{0}}^{\lambda_{1}} d \lambda^{3} g_{r s}\left(\eta_{(i j)}(\lambda, \tau)\right) \frac{\partial \eta_{(i j)}^{r}(\lambda, \tau)}{\partial \lambda} \frac{\partial \eta_{(i j)}^{s}(\lambda, \tau)}{\partial \lambda}$, where $\eta_{(i j)}(\lambda, \tau)$ is the three-geodesic joining $\vec{\eta}_{i}(\tau)$ and $\vec{\eta}_{j}(\tau)$ (see [62]). The momenta are covectors defined at the positions of the particles.

In this case, the distribution function, satisfying the Liouville theorem with the Hamiltonian $\mathcal{M c}$, is:

$$
\begin{aligned}
& \tilde{f}_{(m c)}\left(\vec{\rho}_{1}, . ., \vec{\pi}_{N-1}|\mathcal{E}, \overrightarrow{\mathcal{S}}, V, N| g, g^{r}\right)=\tilde{Z}^{-1}\left(\mathcal{E}, \overrightarrow{\mathcal{S}}, V, N \mid g, g^{r}\right) \chi(V) \delta\left(M_{N}\left(\vec{\eta}_{i}, \vec{\kappa}_{i} ; g, g^{r}\right) c-\mathcal{E}\right) \\
& \delta^{3}\left(J_{N}^{u}\left(\vec{\eta}_{i}, \vec{\kappa}_{i} ; g, g^{r}\right)-\mathcal{S}^{u}\right) \delta^{3}\left(\hat{\mathcal{P}}_{N}^{u}\left(\vec{\eta}_{i}, \vec{\kappa}_{i} ; g, g^{r}\right)\right) \delta^{3}\left(\frac{\hat{\mathcal{K}}_{N}^{u}\left(\vec{\eta}_{i}, \vec{\kappa}_{i} ; g, g, g^{r}\right)}{M_{N}\left(\vec{\eta}_{i}, \hat{\kappa}_{i} ; g^{\prime}, g^{r}\right) c}\right) .
\end{aligned}
$$

Notwithstanding the presence of the time-dependent long-range inertial potentials, one has $\partial_{\tau} f_{(m c)}=0$, because in the non-inertial rest frames, $J_{N}^{u}\left(\vec{\eta}_{i}, \vec{\kappa}_{i} ; g, g^{r}\right), \hat{\mathcal{P}}_{N}^{u}\left(\vec{\eta}_{i}, \vec{\kappa}_{i} ; g, g^{r}\right), \frac{\hat{\mathcal{K}}_{N}^{u}\left(\vec{\eta}_{i}, \vec{\kappa}_{i} ; g, g^{r}\right)}{M_{N}\left(\vec{\eta}_{i}, \vec{\kappa}_{i} ; g, g^{r}\right) c}$ are asymptotic constants of the motion at spatial infinity.

As a consequence, there is a notion of non-inertial equilibrium for the distribution function gauge equivalent to inertial equilibrium in the sense of Section 2, at least in the passive viewpoint where one does not actively accelerate the gas, but one goes passively from an inertial to a non-inertial frame.

\subsection{The Micro-Canonical Ensemble in the Non-Relativistic Non-Inertial Rest Frames}

By using the results of Section 3.1 for the non-inertial Galilei generators, one gets the following definition of the non-relativistic extended partition function in non-inertial rest frames (see Appendix $C$ of [35] for the explicit form for free particles):

$$
\begin{aligned}
\tilde{Z}_{(n r)}\left(\mathcal{E}, \overrightarrow{\mathcal{S}}, V \mid g, g^{r}\right)= & \frac{1}{N !} \int \prod_{i}^{1 . . N} d^{3} \eta_{i} \chi(V) \int \prod_{j}^{1 . . N} d^{3} \kappa_{j} \delta\left(E_{\text {Galilei, },}-\mathcal{E}\right) \\
& \delta^{3}\left(\vec{S}_{\text {Galilei,N }}-\overrightarrow{\mathcal{S}}\right) \delta^{3}\left(\overrightarrow{\mathcal{P}}_{\text {Galilei, }, N}\right) \delta^{3}\left(\frac{\mathcal{\mathcal { K }}_{\text {Gallile }, N}}{m}\right) .
\end{aligned}
$$

For the distribution function, one has:

$$
\begin{aligned}
\tilde{f}_{(m c, n r)}\left(\vec{\rho}_{1}, . ., \vec{\pi}_{N-1}|\mathcal{E}, \overrightarrow{\mathcal{S}}, V, N| g, g^{r}\right)= & \tilde{Z}_{(n r)}^{-1}\left(\mathcal{E}, \overrightarrow{\mathcal{S}}, V, N \mid g, g^{r}\right) \chi(V) \delta\left(E_{\text {Galilei }, N}-\mathcal{E}\right) \\
& \delta^{3}\left(\vec{S}_{\text {Galilei, } N}-\overrightarrow{\mathcal{S}}\right) \delta^{3}\left(\overrightarrow{\mathcal{P}}_{\text {Galilei, } N}\right) \delta^{3}\left(\frac{\overrightarrow{\mathcal{K}}_{\text {Galilei, }}}{m}\right) .
\end{aligned}
$$

By using the Hamilton equations generated by the Hamiltonian $\mathcal{M}_{\text {Galilei }}$ of Section 3.1, it can be checked that it satisfies the Liouville theorem. Since the Galilei generators at spatial infinity are constant, we get again an equilibrium distribution function, $\partial_{t} \tilde{f}_{(m c, n r)}=0$, like in inertial frames.

\section{The Lorentz-Scalar Micro-Canonical Temperature in the Non-Relativistic and Relativistic Inertial Rest Frame}

The micro-canonical entropy in the standard non-relativistic micro-canonical ensemble has the definition:

$$
S_{(m c, n r, s t)}(E, V, N)=\frac{1}{N} \ln \tilde{Z}_{(n r, s t)}(E, V, N),
$$

and $d S_{(m c, n r, s t)} \geq 0$ is the second law of thermodynamics. 
The micro-canonical temperature $T_{(m c)}=T_{(m c)}(E, V, N)$ (see $[62,66,98] ; k_{B}$ is the Boltzmann constant) has the following definition (it is a functional of the inertial potentials):

$$
\frac{1}{k_{B} T_{(m c)}}=\left.\frac{\partial S_{(m c, n r, s t)}(E, V, N)}{\partial E}\right|_{V, N}=\left.\frac{1}{\tilde{Z}_{(n r, s t)}(E, V, N)} \frac{\partial \tilde{Z}_{(n r, s t)}(E, V, N)}{\partial E}\right|_{V, N} .
$$

The equations $\frac{P_{(m c)}}{k_{B} T_{(m c)}}=\left.\frac{\partial S_{(m c}(E, V, N)}{\partial V}\right|_{E, N}$ and $\frac{\mu_{(m c)}}{k_{B} T_{(m c)}}=\left.\frac{\partial S_{(m c}(E, V, N)}{\partial N}\right|_{E, V}$ define the pressure $P_{(m c)}$ and the chemical potential $\mu_{(m c)}$. Then, one has the Gibbs relation $d E=T_{(m c)} d S_{(m c, n r, s t)}-P_{(m c)} d V+$ $\mu_{(m c)} d N$.

These definitions can be adapted to the non-relativistic rest frame and then extended to the relativistic rest frame by replacing the micro-canonical entropy (25) with the entropies $S_{(m c, n r)}(\mathcal{E}, V, N)=\frac{1}{N} \ln \tilde{Z}_{(n r)}(\mathcal{E}, V, N)$ and $S_{(m c)}(\mathcal{E}, V, N)=\frac{1}{N} \ln \tilde{Z}(\mathcal{E}, V, N)$, respectively.

As a consequence, in the relativistic inertial rest frame, the micro-canonical temperature $T_{(m c)}$, defined by $\frac{1}{k_{B} T_{(m c)}}=\left.\frac{1}{\tilde{Z}(\mathcal{E}, V, N)} \frac{\partial \tilde{Z}(\mathcal{E}, V, N)}{\partial \mathcal{E}}\right|_{V, N}$, is a Lorentz scalar, because the relativistic internal energy $\mathcal{E}$ is a Lorentz scalar like the internal energy $M c$, and as said after Equation (19), also the non-dynamical volume $\mathrm{V}$ is a frame independent Lorentz scalar.

Since in the presence of short-range forces, one has the equivalence of the micro-canonical and canonical ensembles, the thermodynamic limit $N, V \rightarrow \infty$ with $N / V=$ const. gives rise to a canonical temperature $T$, limit of $T_{(m c)}(E, V, N)$, which is a Lorentz scalar in the relativistic rest frame instant form of dynamics. With long-range forces, the micro-canonical ensemble is inequivalent to the canonical ensemble (in which there is negative heat capacity), as shown in [37,47-50], and the canonical temperature is not defined starting from the micro-canonical one.

In inertial non-relativistic frames in the standard case of the ideal Boltzmann gas ( $\mathrm{N}$ free non-relativistic particles of mass $m$ and energy $\frac{\vec{p}^{2}}{2 m}$ ), Equations (9) and (26) imply $\frac{1}{k_{B} T_{(m c)}} \rightarrow_{N \rightarrow \infty} \frac{3 N}{2 E}$ (like in the classical virial theorem). Moreover, one gets $p_{(m c)}=\left.k_{B} T_{(m c)} \frac{\partial S_{(m c, n r, s t}(E, V, N)}{\partial V}\right|_{E, N} \rightarrow_{N \rightarrow \infty} k_{B} T_{(m c)} \frac{N}{V}$, and the resulting equation of state is $p_{(m c)} V=N k_{B} T_{(m c)}$. These results can be reproduced also in the non-relativistic inertial rest frame by replacing Equation (9) with Equation (15), as shown in Section 5 of Appendix C of [35]. When the thermodynamics limit is well defined, then $T_{(m c)}$ and $p_{(m c)}$ become the canonical temperature and pressure, respectively.

In the relativistic inertial rest frame, an explicit analytic form of the micro-canonical entropy $S_{(m c)}(\mathcal{E}, V, N)=\frac{1}{N} \ln \tilde{Z}(\mathcal{E}, V, N)$ is not known (see Section 6 of Appendix $C$ of [35]), so that one cannot obtain an explicit equation of state for a relativistic ideal Boltzmann gas ( $\mathrm{N}$ free relativistic particles of mass $m$ and energy $\sqrt{m^{2} c^{2}+\vec{\kappa}^{2}}$ ). However, in [2], by using the equilibrium Jüttner one-particle distribution function, it is shown that also in the relativistic case, one obtains $p=\frac{N}{V} k_{B} T$.

The extension of these results to non-relativistic and relativistic non-inertial frames requires that the definitions in Equations (25) and (26) are still valid with non-inertial motion, and this is an open problem.

\section{The Relativistic One-Particle Distribution Function in the Relativistic Inertial Rest Frames}

As shown in [1] in the non-relativistic kinetic theory of diluted gases, one can introduce the (non-equilibrium) one-particle distribution function:

$$
\begin{aligned}
f(\vec{x}, \vec{p}, t) & =<\sum_{i} \delta^{3}\left(\vec{x}-\vec{x}_{i}\right) \delta^{3}\left(\vec{p}-\vec{p}_{i}\right)>_{(\text {Gibbs })} \\
& =\int \prod_{i=1}^{N} d^{3} x_{i} d^{3} p_{i} \sum_{i} \delta^{3}\left(\vec{x}-\vec{x}_{i}\right) \delta^{3}\left(\vec{p}-\vec{p}_{i}\right) \rho\left(\vec{x}_{1}, \vec{p}_{1}, \ldots, \vec{x}_{N}, \vec{p}_{n}, t\right) \\
& =N \int \prod_{i=2}^{N} d^{3} x_{i} d^{3} p_{i} \rho\left(\vec{x}_{1}, \vec{p}_{1}, \ldots, \vec{x}_{N}, \vec{p}_{n}, t\right),
\end{aligned}
$$


by means of a statistical average on a non-equilibrium Gibbs ensemble. The normalized density function $\rho\left(\vec{x}_{1}, \vec{p}_{1}, \ldots, \vec{x}_{N}, \vec{p}_{n}, t\right)[101]$ is symmetric in the exchange of particles (all equal with mass $\mathrm{m}$ ) and satisfies the Liouville theorem implied by the Hamilton equations for the $\mathrm{N}$ particles with given Hamiltonian $H: \frac{d \rho}{d t}=\frac{\partial \rho}{\partial t}+\sum_{i=1}^{N}\left(\frac{\partial \rho}{\partial \vec{x}_{i}} \cdot \frac{d \vec{x}_{i}}{d t}+\frac{\partial \rho}{\partial \vec{p}_{i}} \cdot \frac{d \vec{p}_{i}}{d t}\right)=0$.

The function $f(\vec{x}, \vec{p}, t)$ satisfies the Boltzmann transport equation ( $\vec{F}$ is an external force):

$$
\left(\frac{\partial}{\partial t}+\frac{\vec{p}}{m} \cdot \frac{\partial}{\partial \vec{x}}+\vec{F} \cdot \frac{\partial}{\partial \vec{p}}\right) f(\vec{x}, \vec{p}, t)=\left(\frac{\partial f}{\partial t}\right)_{\text {coll. }},
$$

where $\left(\frac{\partial f}{\partial t}\right)_{\text {coll. }}$ is the collision term [102]. In absence of external, forces an equilibrium distribution is assumed to be independent from $\vec{x}$ and to satisfy $\frac{\partial}{\partial t} f_{(e q)}=0$. Therefore, it is a solution of the Boltzmann equation $\left(\frac{\partial f_{(e q)}}{\partial t}\right)_{\text {coll. }}=0$.

In the case of free particles, the solution is the Maxwell-Boltzmann distribution function $e^{-\beta \frac{\vec{p}^{2}}{2 m}}$ with $\beta=1 / k_{B} T$ ( $T$ is the canonical temperature).

The Boltzmann equation for $f(\vec{x}, \vec{p}, t)$ can be derived as an approximation starting from the coupled equations of motion (the Bogoliubov-Born-Green-Kirkwood-Yvon (BBGKY) hierarchy; see Chapter 3 of [1]) for the s-particle distribution functions $f_{s}\left(\vec{x}_{1}, \vec{p}_{1}, \ldots, \vec{x}_{s}, \vec{p}_{s}, t\right)=$ $\frac{N !}{(N-s) !} \int \prod_{i=s+1}^{N} d^{3} x_{i} d^{3} p_{i} \rho\left(\vec{x}_{1}, \vec{p}_{1}, \ldots, \vec{x}_{N}, \vec{p}_{n}, t\right)$ by using the Liouville theorem.

Till now, in absence of a consistent RCM for $\mathrm{N}$ interacting particles and with only the relativistic kinetic theory of $[103,104]$, the relativistic Boltzmann equation is postulated [105-109]. However, the transformation properties under Lorentz transformations of the one-particle distribution function satisfying it are an open problem reviewed in [110-115].

In absence of external forces and assuming $p^{o}=\sqrt{m^{2} c^{2}+\vec{p}^{2}}$, the relativistic Boltzmann equation takes the form $p_{\mu} \frac{\partial}{\partial x^{\mu}} f\left(x^{\alpha}, p^{\alpha}\right)=C\left(x^{\alpha}, p^{\alpha}\right)$ with the second member being the collision term.

For free particles, the equilibrium solution of the standard relativistic Boltzmann equation is the equilibrium homogeneous Boltzmann-Jüttner distribution [116-118]:

$$
f_{(e q)}(\vec{x}, \vec{p})=f_{(e q)}(\vec{p})=A e^{-\beta^{\mu} p_{\mu}} \stackrel{\text { rest frame }}{=} A e^{-\beta \sqrt{m^{2}+\vec{p}^{2}}},
$$

where $T$ is the canonical temperature in the rest frame [119] and $A=\frac{\text { const. }}{4 \pi k_{B} T m^{2} c K_{2}\left(\frac{m c^{2}}{k_{B} T}\right)}$. In $[98,110]$ is a discussion of the existing alternatives to the Jüttner distribution and their rebuttal. The non-relativistic limit reproduces the Maxwell-Boltzmann distribution $e^{-\beta \frac{\vec{p}^{2}}{2 m}}$.

In Section 7.1 is a delineation of how to define a Lorentz scalar relativistic one-particle distribution function $f(\vec{\eta}, \vec{\kappa}, \tau)$ in the Wigner three-spaces of the relativistic inertial rest frame for an isolated system of particles in a Lorentz invariant non-dynamical volume.

Then, in Section 7.2 is a sketch of which are the problems in an approach to the relativistic Boltzmann equation when one starts from a relativistic BBGKY hierarchy based on a model for $\mathrm{N}$ interacting relativistic particles.

The extension to non-inertial frames is an open problem.

\subsection{The One-Particle Distribution Function in the Relativistic Inertial Rest Frame}

Let us consider a particle in the Wigner three-space of the inertial rest frame with canonical coordinates $\vec{\eta}(\tau), \vec{\kappa}(\tau)$, whose world-line is $x^{\mu}(\tau)=Y^{\mu}(\tau)+\epsilon_{r}^{\mu}(\vec{h}) \eta^{r}(\tau)$ according to Equation (2).

A relativistic one-particle distribution function in the inertial rest frame can be defined by considering a normalized density function for a relativistic Gibbs ensemble (assumed to transform like the relativistic micro-canonical distribution function to which it tends at equilibrium) of the type $\rho\left(\vec{\eta}_{1}, \vec{\kappa}_{1}, \ldots, \vec{\eta}_{N}, \vec{\kappa}_{N}, \tau\right)$ with $\vec{\eta}_{i}, \vec{\kappa}_{i}, i=1, \ldots, N$, being Wigner three-vectors in the Wigner three-spaces. 
Then, the natural definition for the one-particle distribution function in the Wigner three-spaces without a dependence on both the external and internal centers of mass is (see Equation (8) for $\chi(V)$ ):

$$
\begin{aligned}
f(\vec{\eta}(\tau), \vec{\kappa}(\tau), \tau)= & <\sum_{i} \delta^{3}\left(\vec{\eta}(\tau)-\vec{\eta}_{i}(\tau)\right) \delta^{3}\left(\vec{\kappa}(\tau)-\vec{\kappa}_{i}(\tau)\right)>_{(G i b b s)} \\
= & \int \prod_{j}^{1 . N} d^{3} \eta_{j} \chi(V) \int \prod_{k}^{1 . . N} d^{3} \kappa_{k} \sum_{i} \delta^{3}\left(\vec{\eta}(\tau)-\vec{\eta}_{i}(\tau)\right) \delta^{3}\left(\vec{\kappa}(\tau)-\vec{\kappa}_{i}(\tau)\right) \\
& \rho\left(\vec{\eta}_{1}, \vec{\kappa}_{1}, \ldots, \vec{\eta}_{N}, \vec{\kappa}_{N}, \tau\right) \\
= & \int \prod_{i}^{1 . . N} d^{3} \eta_{i} \chi(V) \int \prod_{j}^{1 . . N} d^{3} \kappa_{j} \rho\left(\vec{\eta}_{1}, \vec{\kappa}_{1}, \ldots, \vec{\eta}_{N}, \vec{\kappa}_{N}, \tau\right) \\
& \delta^{3}\left(\overrightarrow{\mathcal{P}}_{N}\right) \delta^{3}\left(\frac{\overrightarrow{\mathcal{K}}_{N}}{M_{N} c}\right)\left[\sum_{i} \delta^{3}\left(\vec{\eta}(\tau)-\vec{\eta}_{i}(\tau)\right) \delta^{3}\left(\vec{\kappa}(\tau)-\vec{\kappa}_{i}(\tau)\right)\right] .
\end{aligned}
$$

In the case of an equilibrium ensemble, the replacement $\rho\left(\vec{\eta}_{1}, \vec{\kappa}_{1}, \ldots, \vec{\eta}_{N}, \vec{\kappa}_{N}, \tau\right) \mapsto$ $Z^{-1}(\mathcal{E}, V, N) \frac{1}{N !} \delta\left(M_{N} c^{2}-\mathcal{E}\right)$ transforms Equation (30) in a statistical average in the relativistic micro-canonical ensemble of the type of Equation (10). Therefore, the density function depends only on relative variables, $\rho\left(\vec{\eta}_{1}, \vec{\kappa}_{1}, \ldots, \vec{\eta}_{N}, \vec{\kappa}_{N}, \tau\right) \delta^{3}\left(\overrightarrow{\mathcal{P}}_{N}\right) \delta^{3}\left(\frac{\overrightarrow{\mathcal{K}}_{N}}{M_{N} c}\right)=\tilde{\rho}\left(\vec{\rho}_{1}, \vec{\pi}_{1}, \ldots, \vec{\rho}_{N-1}, \vec{\pi}_{N-1}, \tau\right) \delta^{3}\left(\overrightarrow{\mathcal{P}}_{N}\right) \delta^{3}\left(\frac{\overrightarrow{\mathcal{K}}_{N}}{M_{N c} c}\right)$, and has the same transformation properties as the micro-canonical distribution function $f_{(m c)}\left(\vec{\rho}_{1}, . ., \vec{\pi}_{N-1} \mid \mathcal{E}, V, N\right)$ of Equation (21). Equation (30) is a tentative ansatz for a non-equilibrium relativistic configuration in a Lorentz scalar volume whose implications are an open problem.

By using Equation (2.27) of [35], we can rewrite the internal Poincaré generators in terms of $\vec{x}_{(c m) i}\left(\tau=x_{(c m)}^{o}\right)$ and $\vec{p}_{(c m) i}\left(\tau=x_{(c m)}^{o}\right)$, and we have $d^{3} \eta_{i} d^{3} \kappa_{i}=d^{3} x_{(c m) i} d^{3} p_{(c m) i}$. Since the density function depends only on the relative variables, the dependence on the non-covariant Jacobi data $\vec{z}_{(\mathrm{cm})}$ of the external center of mass drops out. Therefore, Equation (30) can be rewritten in the Cartesian coordinates of the rest frame in the following form:

$$
\begin{aligned}
f(\vec{\eta}(\tau), \overrightarrow{\mathcal{\kappa}}(\tau), \tau)= & \int \prod_{i}^{1 . N} d^{3} \eta_{i} \chi(V) \int \prod_{j}^{1 . . N} d^{3} \kappa_{j} \\
= & \rho\left(\vec{\eta}_{1}, \vec{\kappa}_{1}, \ldots, \vec{\eta}_{N}, \vec{\kappa}_{N}, \tau\right) \delta^{3}\left(\overrightarrow{\mathcal{P}}_{N}\right) \delta^{3}\left(\overrightarrow{\mathcal{P}}_{N}\right) \delta^{3}\left(\frac{\overrightarrow{\mathcal{K}}_{N}}{\left.M_{N c}\right)}\right. \\
= & {\left[\sum_{i} \delta^{3}\left(\vec{x}\left(\tau=x_{(c m)}^{o}\right)-\vec{x}_{(c m) i}\left(\tau=x_{(c m)}^{o}\right)\right)\right.} \\
& \left.\delta^{3}\left(\vec{p}\left(\tau=x_{(c m)}^{o}\right)-\vec{p}_{(c m) i}\left(\tau=x_{(c m)}^{o}\right)\right)\right] \\
= & F_{(c m)}\left(x_{(c m)}^{o}, \vec{x}_{(c m)}\left(x_{(c m)}^{o}\right), \vec{p}_{(c m)}\left(x_{(c m)}^{o}\right)\right) .
\end{aligned}
$$

In the free case, one can use the results of Subsection IIC of [35] to rewrite the one-particle distribution function in an arbitrary inertial frame with Cartesian coordinates $x^{\mu}=\Lambda^{\mu}{ }_{v} x_{(\mathrm{cm})}^{v}$ and to show explicitly that it is a Lorentz scalar.

In the relativistic rest frame in absence of the external relativistic center of mass and at least for $N, V \rightarrow \infty$ with $N / V=$ const., the analogue of the equilibrium homogeneous Boltzmann-Jüttner distribution for free particles is expected to be [120] $f_{(e q)}(\vec{\eta}, \vec{\kappa})=A e^{-\frac{\sqrt{m^{2} c^{2}+\vec{k}^{2}}}{k_{B} T}}$.

\subsection{The Open Problem of the Relativistic Boltzmann Equation}

The method of the BBGKY hierarchy, used in Chapter 3 of [1] to derive the non-relativistic Boltzmann equation by using the Liouville equation, does not work in the relativistic case with action-at-a-distance interactions. This is due to the presence of the potentials under the energy square roots and moreover due to the momentum dependence of the potentials as said at the end of Section 2.1 and as shown explicitly [121] in Section 4 of [35], where there is not only the explicit form of the internal Poincaré generators for $\mathrm{N}$ scalar particles with action-at-a-distance interactions, but also the expression of the Liouville operators acting on the single particles.

In the Wigner three-spaces of the relativistic inertial rest frame after the elimination of the external center of mass, the $\mathrm{N}$ scalar particles in the RCM of Section 2 are described by Wigner-covariant phase space three-variables $\vec{\eta}_{i}(\tau), \vec{\kappa}_{i}(\tau), i=1, . ., N$, (or by the relative ones $\vec{\rho}_{a}(\tau), \vec{\pi}_{a}(\tau), a=1, \ldots, N-1$, after the elimination of the internal center of mass in the Wigner three-spaces). 
Then, one can introduce not only the one-particle distribution function $f(\vec{\eta}(\tau), \vec{\kappa}(\tau), \tau)$ of Equation (30), but also the s-particle distribution (or correlation) functions $f_{s}\left(\vec{\eta}_{1}, \vec{\kappa}_{1}, . ., \vec{\eta}_{s}, \vec{\kappa}_{s}, \tau\right)$, $s=1, \ldots, N$, in the usual way [122]:

$$
\begin{aligned}
f(\vec{\eta}(\tau), \vec{\kappa}(\tau), \tau)= & f_{1}(\vec{\eta}(\tau), \vec{\kappa}(\tau), \tau) \\
= & N \int \prod_{j}^{2 . N} d^{3} \eta_{j} \chi(V) \int \prod_{k}^{2 . N} d^{3} \kappa_{k} \rho\left(\vec{\eta}(\tau), \vec{\kappa}(\tau), \vec{\eta}_{2}(\tau) . ., \vec{\kappa}_{N}(\tau), \tau\right), \\
f_{s}\left(\vec{\eta}_{1}(\tau), \vec{\kappa}_{1}(\tau), . ., \vec{\eta}_{s}(\tau), \vec{\kappa}_{s}(\tau), \tau\right)= & \frac{N !}{(N-s) !} \int \prod_{j}^{s+1 . N} d^{3} \eta_{j} \chi(V) \int \prod_{k}^{s+1 . N} d^{3} \kappa_{k} \\
& \rho\left(\vec{\eta}_{1}(\tau), \vec{k}_{1}(\tau), . ., \vec{\eta}_{s}(\tau), \vec{k}_{s}(\tau) \mid \vec{\eta}_{s+1}(\tau), \ldots, \vec{\kappa}_{N}(\tau), \tau\right) .
\end{aligned}
$$

The Liouville operator implied by the Hamiltonian $M c=\sum_{i} E_{i}$ (the global invariant mass) of Section 2.1 with $E_{i}=\sqrt{m_{i}^{2} c^{2}+\vec{\kappa}_{i}^{2}(\tau)+V_{i}(\tau)}$ and $V_{i}(\tau)=\tilde{V}_{i}\left(\vec{\eta}_{m}(\tau)-\vec{\eta}_{n}(\tau), \vec{\kappa}_{i}(\tau)\right)$, is:

$$
\begin{aligned}
\hat{L} & =\frac{\partial}{\partial \tau}+\hat{h}_{N}, \\
\hat{h}_{N} & =\sum_{i}^{1 \ldots N}\left[\left(\frac{\kappa_{i r}(\tau)}{E_{i}(\tau)}+\sum_{j} \frac{1}{2 E_{j}(\tau)} \frac{\partial V_{j}(\tau)}{\partial \kappa_{i r}}\right) \frac{\partial}{\partial \eta_{i}^{r}}-\sum_{j} \frac{1}{E_{j}(\tau)} \frac{\partial V_{j}(\tau)}{\partial \eta_{i}^{r}} \frac{\partial}{\partial \kappa_{i r}}\right] .
\end{aligned}
$$

This implies the following coupled equations of motion for the distribution functions $\left(d z_{i}=d^{3} \eta_{i} \chi(V) d^{3} \kappa_{i} ; \hat{L} \rho=0\right)$ :

$$
\begin{aligned}
& \frac{\partial}{\partial \tau} f_{s}\left(\vec{\eta}_{1}(\tau), \vec{\kappa}_{1}(\tau), . ., \vec{\eta}_{s}(\tau), \vec{\kappa}_{s}(\tau), \tau\right) \\
= & \frac{N !}{(N-s) !} \int d z_{s+1} \ldots d z_{N} \frac{\partial}{\partial \tau} \rho(1, \ldots, N, \tau) \\
= & -\frac{N !}{(N-s) !} \int d z_{s+1} \ldots d z_{N} \hat{h}_{N} \rho(1, \ldots, N, \tau) .
\end{aligned}
$$

However, these equations do not produce a relativistic BBGKY hierarchy, from which the relativistic Boltzmann equation implied by the RCM of Section 2 with a decoupled external relativistic center of mass could be defined in absence of external forces. This is due to the fact that due to the potentials and momenta inside $E_{i}(\tau)$, differently from the non-relativistic case (where $\hat{h}_{N}=\sum_{i=1}^{N} \frac{\vec{\kappa}_{i}}{m}$. $\left.\frac{\partial}{\partial \vec{\eta}_{i}}+\frac{1}{2} \sum_{i \neq j} \hat{P}_{i j}\right)$, one does not have $\hat{h}_{N}(1, . ., N)=\hat{h}_{s}(1, . . s)+\hat{h}_{N-s}(s+1, . ., N)+\sum_{u=1}^{s} \sum_{v=s+1}^{N} \hat{P}_{u v}$ (with $\left.\hat{P}_{i j}=\vec{f}\left(\left|\vec{\eta}_{i}-\vec{\eta}_{j}\right|\right) \cdot\left(\frac{\partial}{\partial \vec{k}_{i}}-\frac{\partial}{\partial \vec{k}_{j}}\right)\right)$ and $\int d z_{s+1} \ldots . d z_{N} \hat{h}_{N-s}(s+1, \ldots, N) \rho(1, \ldots, N, \tau)=0$.

Therefore, one does not get $\left(\frac{\partial}{\partial \tau}+\hat{h}_{s}\right) f_{s}=\ldots . . \sum_{j=1}^{s} \int d z_{s+1} \hat{P}_{i, s+1} f_{s+1}(1, . ., s+1, \tau)$, namely the basic equation (see Equation (3.57) of [1]) for defining the BBGKY hierarchy.

As a consequence, a relativistic Boltzmann equation emerging from this framework is expected to be more complex than the standard form of [105-108], and one would have to see whether the relativistic Jüttner distribution function still emerges in this RCM framework after the decoupling of the external center of mass. The solution of this problem is one of the main open problems in relativistic statistical mechanics.

\section{Relativistic Dissipative Fluids}

The moments of the one-particle distribution function, the solution of the Boltzmann equation, give rise to a hydrodynamical description of relativistic kinetic theory by means of an effective dissipative fluid (to be contrasted with the perfect or dissipative fluids of relativistic hydrodynamics).

This allows one to study the problems of relativistic dissipative fluids and of causal relativistic thermodynamics, whose foundations are not yet fully established (see Endnote [123] — which refers to References [124-126], but which is used for instance as a hydrodynamical model for describing relativistic heavy-ion collisions [127-130]. See [131-134] for a review of the Eckart, Landau-Lifschitz, Israel-Stewart [103] and Carter [135] points of view and [136] for the $1+3$ point of view. 
The various approaches differ in the parametrization of the relativistic entropy current $S^{\mu}=s u^{\mu}+q^{\mu}+V^{\mu}$ in terms of the fluid four-velocity $u^{\mu}$, the ordinary entropy $s$, the heat transfer four-vector $q^{\mu}$ and the viscous terms $V^{\mu}$ (with $q^{\mu}$ and $V^{\mu}$ orthogonal to $u^{\mu}$ ), in the definition of $u^{\mu}$ and in the order of the deviations from equilibrium (linear for the Eckart and Landau-Lifschitz models, quadratic for the Israel-Stewart and Carter models). See [104] and the Appendix B of [137] for reviews; for recent developments, see [138-142]. Let us remark that this entropy current is postulated without saying which type of local equilibrium is presupposed and whether it can be applied to the isolated systems in non-dynamical volumes discussed in the previous sections.

Then, one has to implement the second law of thermodynamics in the form $\partial_{\mu} S^{\mu} \geq 0$. In Carter's two-fluid approach, one writes $S^{\mu}=s u^{\mu}+j^{\mu}$ and considers $j^{\mu}$ as a second fluid; a Lagrangian-like approach is used to implement $\partial_{\mu} S^{\mu} \geq 0$. As shown in [143], the Israel-Stewart and Carter approaches are essentially equivalent.

What is lacking in all of these approaches is a variational principle describing relativistic fluids out of equilibrium and implying $\partial_{\mu} S^{\mu} \geq 0$.

In Section 8.1 is a sketch of the standard approach with the existing relativistic mechanics.

In Section 8.2 is a sketch of how one could face these problems in the relativistic inertial rest frame by using an action principle for relativistic fluids derived by the one of [144].

\subsection{The Standard Approach with Relativistic Statistical Mechanics}

In equilibrium relativistic statistical mechanics [103], a perfect particle fluid with internal energy $\rho$ and pressure $p$ (connected by some equation of state) has an energy-momentum tensor $T^{\mu v}=\epsilon \rho U^{\mu} U^{v}-p\left(\eta^{\mu v}-\epsilon U^{\mu} U^{v}\right)$, where the unit hydrodynamical four-velocity $U^{\mu}$ is a time-like eigenvector of the energy-momentum tensor, $\epsilon U_{\mu} T^{\mu v}=\rho U^{v}$. The conserved particle current is $J^{\mu}=n U^{\mu}$ with $n$ the particle number density. All of the relevant four-vectors are parallel to $U^{\mu}$ at equilibrium: $P^{\mu}=p U^{\mu}, V^{\mu}=V U^{\mu}, \beta^{\mu}=\beta U^{\mu}\left(\beta=1 / k_{B} T\right)$. In relativistic thermodynamics, also the entropy is a four-vector parallel to $U^{\mu}: S^{\mu}=S U^{\mu}=\frac{p}{c^{2}} \beta^{\mu}-\alpha J^{\mu}-\epsilon \beta_{v} T^{\nu \mu}\left(\alpha=\beta\left(\mu-k_{B} T S / n\right)\right.$ is the thermal potential with $\mu$ the chemical potential). At equilibrium, one has the Killing equation $\partial_{\mu} \alpha=\partial_{\mu} \beta_{v}+\partial_{\nu} \beta_{\mu}=0$.

As shown in $[103,104]$, near equilibrium, all of these four-vector quantities are no longer parallel with $U^{\mu}$, and $T^{\mu v}$ has the decomposition of a viscous particle fluid. To treat dissipative fluids out of equilibrium, one needs a relativistic kinetic theory, in which the one-particle distribution function of the diluted gas is used to obtain a hydrodynamical description of an effective fluid.

In the existing relativistic kinetic theory, the one-particle distribution function $f(x, p)=\tilde{f}\left(\vec{x}, \vec{p}, x^{o}\right)$, with the particle energy given by $p^{o}=\sqrt{m^{2} c^{2}+\vec{p}^{2}}$, is used to define:

(a) a conserved particle current:

$$
J^{\mu}(x)=\int \frac{m c}{h^{3}} \frac{d^{3} p}{p^{0}} \frac{p^{\mu}}{m c} f(x, p) ;
$$

(b) an energy-momentum tensor [145]:

$$
T^{\mu v}(x)=\int \frac{m c}{h^{3}} \frac{d^{3} p}{p^{0}} \frac{p^{\mu}}{m c} p^{v} f(x, p), \text { with } \epsilon p^{2}=m^{2} c^{2}
$$

(c) an infinite number of higher moments $F^{\mu v \alpha \beta . .}(x)=\int \frac{m c}{h^{3}} \frac{d^{3} p}{p^{0}} \frac{p^{\mu}}{m c} p^{v} p^{\alpha} p^{\beta} \ldots f(x, p)$ (in some approximate model, one gets a closed description only in terms of 14 of them; see [146]).

It is assumed that this is the description of a perfect gas with some equation of state like the Boltzmann perfect gas.

Then, one defines an entropy four-vector: 


$$
S^{\mu}(x)=-k_{B} \int \frac{m c}{h^{3}} \frac{d^{3} p}{p^{o}} \frac{p^{\mu}}{m c} f(x, p) \ln f(x, p) .
$$

and the second law of thermodynamics $\partial_{\mu} S^{\mu}(x) \geq 0$ (H-theorem) emerges.

\subsection{The Approach with the Wigner Three-Spaces of the Relativistic Inertial Rest Frame}

Equilibrium perfect fluids with equation of state $\rho=\rho(n, s)$ ( $n$ is the particle number density, $s$ the equilibrium entropy) can also be described by means of the action principle of [144], which has been adapted to the framework of the RCM of Section 2 in $[137,147,148]$. In this approach, the isentropic fluid is described in terms of Lagrangian comoving three-coordinates $\alpha^{i}(\tau, \vec{\sigma})$ on the Wigner three-spaces of the relativistic inertial rest frame, and the action is:

$$
S=\int d \tau d^{3} \sigma \rho\left(n\left(\alpha^{i}\right), s\left(\alpha^{i}\right)\right)(\tau, \vec{\sigma}) .
$$

The particle number density has the form $n\left(\alpha^{i}(\tau, \vec{\sigma})\right)=\sqrt{\epsilon \eta_{A B} J^{A}\left(\alpha^{i}(\tau, \vec{\sigma})\right) J^{B}\left(\alpha^{i}(\tau, \vec{\sigma})\right)}$, and the unit four-velocity is $U^{A}\left(\alpha^{i}(\tau, \vec{\sigma})\right)=J^{A}\left(\alpha^{i}(\tau, \vec{\sigma})\right) / n\left(\alpha^{i}(\tau, \vec{\sigma})\right)$. It satisfies the comoving condition $U^{A}\left(\alpha^{i}(\tau, \vec{\sigma})\right) \partial_{A} \alpha^{i}(\tau, \vec{\sigma})=0$. This implies the conservation of the particle number, $\partial_{A} J^{A}\left(\alpha^{i}(\tau, \vec{\sigma})\right)=\partial_{A}\left(n\left(\alpha^{i}(\tau, \vec{\sigma})\right) U^{A}\left(\alpha^{i}(\tau, \vec{\sigma})\right)\right)=0$ and the conservation of entropy (no entropy exchange between different fluid particles), $\partial_{A}\left(s\left(\alpha^{i}(\tau, \vec{\sigma})\right) n\left(\alpha^{i}(\tau, \vec{\sigma})\right) U^{A}\left(\alpha^{i}(\tau, \vec{\sigma})\right)\right)=0$, i.e., $U^{A}\left(\alpha^{i}(\tau, \vec{\sigma})\right) \partial_{A} s\left(\alpha^{i}(\tau, \vec{\sigma})\right)=0$.

As shown in [144], this description is in accord with standard relativistic thermodynamics: one has the Euler relation $\rho+p=T s+\mu n$ ( $\mu$ is the chemical potential), the Gibbs relation (first law of thermodynamics) $d \rho=\mu d n+n T d s$ and the second law of thermodynamics at equilibrium, i.e., $U^{A}\left(\alpha^{i}(\tau, \vec{\sigma})\right) \partial_{A} s\left(\alpha^{i}(\tau, \vec{\sigma})\right)=0$.

The RCM framework of Section 2 and Equation (38) suggest that there is the possibility (to be explored) to describe relativistic dissipative fluids with an action principle by relaxing the condition that the fluid is isentropic (see [149] for what is known about non-isentropic fluids).

If one replaces the equilibrium entropy $s\left(\alpha^{i}(\tau, \vec{\sigma})\right)$ with a function $\tilde{s}=\sqrt{\epsilon \eta_{A B} \mathcal{S}^{A} \mathcal{S}^{B}}$ built in terms of a four-vector $\mathcal{S}^{A}\left(\alpha^{i}, \partial_{r} \alpha^{i}, \partial_{r} \partial_{s} \alpha^{i}, ..\right)(\tau, \vec{\sigma})$, we loose the equilibrium condition, i.e., $U^{A}\left(\alpha^{i}(\tau, \vec{\sigma})\right) \partial_{A} \tilde{s}\left(\alpha^{i}(\tau, \vec{\sigma}), \partial_{r} \alpha^{i}(\tau, \vec{\sigma}), ..\right) \neq 0$ due to an entropy exchange among different fluid particles. Following the quoted existing literature on dissipative systems, the four-vector $\mathcal{S}^{A}$ is assumed to have a parametrization of the type:

$$
\mathcal{S}^{A}=s U^{A}+\mathcal{V}^{A}, \quad U_{A} \mathcal{V}^{A}=0,
$$

with $\mathcal{V}^{A}=q^{A}+$ viscous terms ( $q^{A}$ is the heat transfer four-vector).

The new action principle is:

$$
\begin{aligned}
S & =\int d \tau d^{3} \sigma\left[\rho\left(n\left(\alpha^{i}\right), \tilde{s}\left(\alpha^{i}, \partial_{r} \alpha^{i}, . .\right)\right)(\tau, \vec{\sigma})\right. \\
& \left.+\lambda(\tau, \vec{\sigma})\left(\partial_{A} \mathcal{S}^{A}-\sum_{k} \mathcal{A}_{k}^{2}\left(\mathcal{V}^{B}\right)\right)(\tau, \vec{\sigma})\right],
\end{aligned}
$$

with $\lambda(\tau, \vec{\sigma})$ a Lagrange multiplier implying the second law of thermodynamics, $\partial_{A} \mathcal{S}^{A}(\tau, \vec{\sigma}) \geq 0$.

The functions $\mathcal{A}_{k}^{2}\left(\mathcal{V}^{B}\right)(\tau, \vec{\sigma})$ can be taken to have the form resulting from the structure of $\partial_{A} \mathcal{S}^{A}(\tau, \vec{\sigma})$ in the models existing in the literature (like the Eckart or the Israel-Steward models). In this way, one could have both a Lagrangian and Hamiltonian formulation of the equations of motion of dissipative systems and study the difference between the cases in which the Lagrange multiplier remains arbitrary (first-class constraints at the Hamiltonian level) and those in which it turns out to be determined by the dynamics (second-class constraints at the Hamiltonian level). In some cases, it could also happen that the assumed functions $\mathcal{A}_{k}^{2}\left(\mathcal{V}^{B}\right)(\tau, \vec{\sigma})$ are incompatible with the dynamics leading to contradictions. These problems have still to be studied. 
Finally, if one would try to extend this formulation to the non-inertial rest frames, one should use the unit normal to the three-spaces $l^{\mu}(\tau, \vec{\sigma})$ as a unit four-velocity (the congruence of the time-like Eulerian observers). This would be an alternative to both "Eckart theory" (four-velocity parallel to the particle current [150]) and "Landau-Lifshitz theory" (four-velocity as the time-like eigenvector of the energy-momentum tensor).

\section{Conclusions}

The new RCM described in Section 2 allows a reformulation of relativistic kinetic theory and relativistic statistical mechanics of isolated systems with any kind of interactions in the relativistic inertial rest frames after the elimination of the canonical non-covariant (non-local and therefore, non-measurable) center of mass. This introduces a spatial non-separability implying that only Wigner-covariant relative variables inside the Wigner three-spaces have to be used for the description of the isolated system. Moreover, this framework can be extended to the relativistic non-inertial rest frames, whose non-relativistic limit defines the non-relativistic non-inertial rest frames.

As a consequence, for the first time, it is possible to define the relativistic micro-canonical ensemble in both inertial and non-inertial relativistic rest frames of arbitrary isolated systems staying in a non-dynamical Lorentz invariant volume and to show that the micro-canonical temperature is a Lorentz scalar. The non-relativistic limit allows one to get the not yet existing description of such an ensemble in non-relativistic non-inertial frames.

This new framework allows one to begin to face the problem of how to arrive at a relativistic Boltzmann equation starting with the relativistic coupled equations for the s-particle correlation functions of this type of isolated system, which does not allow one to define a trivial BBGKY hierarchy like in the non-relativistic case.

Finally, the description of fluids in the relativistic inertial rest frame can be used to begin to define a new hydrodynamical description of relativistic kinetic theory so as to arrive at an understanding of what type of local equilibrium has to be presupposed in this relativistic description.

The main open problem is the formulation of the relativistic kinetic theory and relativistic statistical mechanics of physical systems in the framework of Einstein general relativity. In the family of globally-hyperbolic, asymptotically-Minkowskian space-times without super-translations discussed in [76-83], there is the possibility of defining the asymptotic ADM Poincare generators at spatial infinity, where the instantaneous three-spaces $\Sigma_{\tau}$ become Euclidean, for every kind of matter. The absence of super-translations implies that these three-spaces are relativistic non-inertial rest frames. To define a micro-canonical ensemble like in Equations (21) and in [43], one has to suppose that all of the matter is contained in a non-dynamical volume $\mathrm{V}$ and that the gravitational field is negligible outside this volume, so that the ten Poincaré generators inside $\mathrm{V}$ can be approximated with the asymptotic ADM ones.

Conflicts of Interest: The author declares no conflict of interest.

\section{References and Notes}

1. Huang, K. Statistical Mechanics; Wiley: New York, NY, USA, 1987.

2. De Groot, S.R.; van Leeuwen, W.A.; van Weert, C.G. Relativistic Kinetic Theory. Principles and Applications; North-Holland: Amsterdam, The Netherlands, 1980.

3. They are called relativistic predictive coordinates by Bell; see [4] and its bibliography.

4. Bel, L.; Martin, J. Predictive Relativistic Mechanics of N Particles with Spin. Annales de l'Institut Henri Poincaré A 1980, 33, 409-442.

5. Dirac, P.A.M. Lectures on Quantum Mechanics; Belfer Graduate School of Science, Monographs Series; Yeshiva University: New York, NY, USA, 1964.

6. Currie, D.G.; Jordan, T.F.; Sudarshan, E.C.G. Relativistic Invariance and Hamiltonian Theories of Interacting Particles. Rev. Mod. Phys. 1965, 35, 350-375. 
7. Lusanna, L. The Chronogeometrical Structure of Special and General Relativity: Towards a BackgroundIndependent Description of the Gravitational Field and Elementary Particles. In General Relativity Research Trends; Reiner, A., Ed.; Nova Science: New York, NY, USA, 2005; Volume 249. arXiv:gr-qc/0404122.

8. Lusanna, L. From Clock Synchronization to Dark Matter as a Relativistic Inertial Effect. In Black Objects in Supergravity; Springer: Berlin/Heidelberg, Germany, 2013; Volume 144, pp. 267-343. arXiv:1205.2481.

9. Steward, J.M. Non-Equilibrium Relativistic Kinetic Theory; Springer: Berlin/Heidelberg, Germany, 1971.

10. Balescu, R. Relativistic Statistical Thermodynamics. Physica 1968, 40, 309-338.

11. Liboff, R.L. Kinetic Theory: Classical, Quantum and Relativistic Descriptions, 2nd ed.; Wiley: New York, NY, USA, 1998.

12. Hakim, R. Introduction to Relativistic Statistical Mechanics: Classical and Quantum; World Scientific: Singapore, 2011.

13. Hakim, R. Remarks on Relativistic Statistical Mechanics I. J. Math. Phys. 1967, 8, 1315.

14. Hakim, R.; Mangeney, A. Relativistic Kinetic Equations Includin Radiation Effects, I. Vlasov Approximation. J. Math. Phys. 1968, 9, 116.

15. Lehmann, E. Covariant Equilibrium Statistical Mechanics. J. Math. Phys. 2006, 47, 023303. arXiv:math-ph/0602060.

16. Horwitz, L.P.; Schieve, W.C.; Piron, C. Gibbs Ensembles in Relativistic Classical and Quantum Mechanics. Ann. Phys. 1981, 137, 306-340.

17. Horwitz, L.P.; Shashoua, S.; Schieve, W.C. A Manifestly Covariant Relativistic Boltzmann Equation for the Evolution of a System of Events. Physica 1989, 161, 300-338.

18. Ares de Parga, G.; López-Carrera, B. Relativistic Statistical Mechanics vs. Relativistic Thermodynamics. Entropy 2011, 13, 1664-1693.

19. Lusanna, L. The N- and 1-Time Classical Descriptions of N-Body Relativistic Kinematics and the Electromagnetic Interaction. Int. J. Mod. Phys. 1997, 12, 645-722.

20. It turns out that the three relevant definitions of the relativistic center of mass-(a) the non-covariant canonical four-center of mass $\tilde{x}^{\mu}(\tau)$, (b) the covariant non-canonical Fokker-Pryce center of inertia $Y^{\mu}(\tau)$ and (c) the non-covariant non-canonical Möller center of energy $R^{\mu}(\tau)$ —can be reformulated as three well-defined functions of the Poincaré generators of the isolated system: therefore, they know the whole instantaneous three-space. This means that the notion of the relativistic center of mass is non-local, and therefore, it is a non-measurable quantity for local measurement apparatuses. Like for the wave function of the universe, who will observe it?

21. Alba, D.; Lusanna, L.; Pauri, M. New Directions in Non-Relativistic and Relativistic Rotational and Multipole Kinematics for N-Body and Continuous Systems. In Atomic and Molecular Clusters: New Research; Ping, Y.L., Ed.; Nova Science: New York, NY, USA, 2006. arXiv:hep-th/0505005.

22. Alba, D.; Lusanna, L.; Pauri, M. Centers of Mass and Rotational Kinematics for the Relativistic N-Body Problem in the Rest-Frame Instant Form. J. Math. Phys. 2002, 43, 1677-1727. arXiv:hep-th/0102087.

23. Alba, D.; Lusanna, L.; Pauri, M. Multipolar Expansions for Closed and Open Systems of Relativistic Particles. J. Math. Phys. 2004, 46, 062505. arXiv:hep-th/0402181.

24. Alba, D.; Crater, H.W.; Lusanna, L. Hamiltonian Relativistic Two-Body Problem: Center of Mass and Orbit Reconstruction. J. Phys. 2007, 40, 9585-9608. arXiv:gr-qc/0610200.

25. Alba, D.; Lusanna, L. Charged Particles and the Electro-Magnetic Field in Non-Inertial Frames: I. Admissible $3+1$ Splittings of Minkowski Spacetime and the Non-Inertial Rest Frames. Int. J. Geom. Methods Phys. 2010, 7, 33-93. arXiv:0908.0213.

26. Alba, D.; Lusanna, L. Charged Particles and the Electro-Magnetic Field in Non-Inertial Frames: II. Applications: Rotating Frames, Sagnac Effect, Faraday Rotation, Wrap-up Effect. Int. J. Geom. Methods Phys. 2010, 7, 185-213. arXiv:0908.0215.

27. Alba, D.; Crater, H.W.; Lusanna, L. Towards Relativistic Atom Physics. I. The Rest-Frame Instant Form of Dynamics and a Canonical Transformation for a system of Charged Particles plus the Electro-Magnetic Field. Can. J. Phys. 2010, 88, 379-424. arXiv:0806.2383.

28. Alba, D.; Crater, H.W.; Lusanna, L. Towards Relativistic Atom Physics. II. Collective and Relative Relativistic Variables for a System of Charged Particles plus the Electro-Magnetic Field. Can. J. Phys. 2010, 88, 425-463. arXiv:0811.0715.

29. Crater, H.W.; Lusanna, L. The Rest-Frame Darwin Potential from the Lienard-Wiechert Solution in the Radiation Gauge. Ann. Phys. 2001, 289, 87-177. 
30. Alba, D.; Crater, H.W.; Lusanna, L. The Semiclassical Relativistic Darwin Potential for Spinning Particles in the Rest Frame Instant Form: Two-Body Bound States with Spin 1/2 Constituents. Int. J. Mod. Phys. 2001, 16, 3365-3478. arXiv:hep-th/0103109.

31. Alba, D.; Crater, H.W.; Lusanna, L. Relativistic Quantum Mechanics and Relativistic Entanglement in the Rest-Frame Instant Form of Dynamics. J. Math. Phys. 2011, 52, 062301. arXiv:0907.1816.

32. Alba, D.; Lusanna, L. Quantum Mechanics in Noninertial Frames with a Multitemporal Quantization Scheme: I. Relativistic Particles. Int. J. Mod. Phys. 2006, 21, 2781-2852. arXiv:hep-th/0502194.

33. Alba, D. Quantum Mechanics in Noninertial Frames with a Multitemporal Quantization Scheme: II. Nonrelativistic Particles. Int. J. Mod. Phys. 2006, 21, 3917-3946. arXiv:hep-th/0504060.

34. Alba, D.; Crater, H.W.; Lusanna, L. On the Relativistic Micro-Canonical Ensemble and Relativistic Kinetic Theory for N Relativistic Particles in Inertial and Non-Inertial Rest Frames. Int. J. Geom. Methods Mod. Phys. 2015, 12, 1550049. arXiv:1202.4667, version 2.

35. Alba, D.; Crater, H.W.; Lusanna, L. On the Relativistic Micro-Canonical Ensemble and Relativistic Kinetic Theory for N Relativistic Particles in Inertial and Non-Inertial Rest Frames. Int. J. Geom. Methods Mod. Phys. 2015, 12, 1550049. arXiv:1202.4667, version 1.

36. For the problems in the definition of the micro-canonical temperature, see [37-41] in the presence of short-range forces and [42] for the case of long-range forces.

37. Rickayzen, G.; Powles, J.G. Temperature in the Classical Microcanonical Ensemble. J. Chem. Phys. 2001, 114, 4333.

38. Rugh, H.H. Dynamical Approach to Temperature. Phys. Rev. Lett. 1997, 78, 772-774.

39. Rugh, H.H. A Geometric, Dynamical Approach to Thermodynamics. J. Phys. 1998, 31, 7761. arXiv:chao-dyn/9703013.

40. Andersen, J.U.; Bonderup, E.; Hansen, K. On the Concept of Temperature for a Small Isolated System. J. Chem. Phys. 2001, 114, 6518.

41. Belega, E.D.; Cheremukhin, E.A.; Elyutin, P.V.; Trubnikov, D.N. On the Definition of the Microcanonical Temperature of Small Weakly Bound Molecular Clusters. Chem. Phys. Lett. 2010, 496, 167-171.

42. Giardiná, C.; Livi, R. Ergodic Properties of Microcanonical Observables. J. Stat. Phys. 1998, 91, 1027-1045.

43. Votyakov, E.V.; Hidmi, H.I.; DeMartino, A.; Gross, D.H.E. Microcanonical Mean-Field Thermodynamics of Self-Gravitating and Rotating Systems. Phys. Rev. Lett. 2002, 89, 031101.

44. It depends not only on the energy of the isolated system, but also on its conserved angular momentum. See [45] for the case of the ideal relativistic quantum gas in relativistic inertial frames.

45. Becattini, F.; Ferroni, L. The Microcanonical Ensemble of the Ideal Relativistic Quantum Gas with Angular Momentum Conservation. Eur. Phys. J. 2007, 52, 597-615.

46. See $[37,47-50]$ for the non-equivalence of micro-canonical and canonical ensembles when long-range forces are present.

47. Campa, A.; Dauxois, T.; Ruffo, S. Statistical Mechanics and Dynamics of Solvable Models with Long-Range Interactions. Phys. Rep. 2009, 480, 57-159. arXiv:0907.0323.

48. Campa, A.; Dauxois, T.; Fanelli, D.; Ruffo, S. Physics of Long-Range Interacting Systems; Oxford University Press: London, UK, 2014.

49. Rocha Filho, T.M.; Amato, M.A.; Figueiredo, A. A Novel Approach to the Determination of Equilibrium Properties of Classical Hamiltonian Systems with Long-Range Interactions. J. Phys. 2009, 42, 165001.

50. Touchette, H. The Large Deviation Approach to Statistical Mechanics. Phys. Rep. 2009, 478, 1-69. arXiv:0804.0327.

51. See [52-61] for the debate on the three possible transformation properties of the temperature under Lorentz transformations and for the existing definitions of relativistic thermodynamics $\left(T=T_{\text {rest }}, T=T_{\text {rest }}(1-\right.$ $\left.\left.v^{2} / c^{2}\right)^{1 / 2}, T=T_{\text {rest }}\left(1-v^{2} / c^{2}\right)^{-1 / 2}\right)$.

52. Van Kampen, N.G. Relativistic Thermodynamics of Moving Systems. Phys. Rev. 1968, 173, 295.

53. Bíró, T.S.; Ván, P. About the Temperature of Moving Bodies. Europhys. Lett. 2010, 89, 30001.

54. Mi, D.; Zhong, H.Y.; Tong, D.M. The Existing Different Proposals for Relativistic Temperature Transformations: The Whys and Wherefores. Mod. Phys. Lett. 2009, 24, 73.

55. Wu, Z.C. Inverse Temperature 4-Vector in Special Relativity. Europhys. Lett. 2009, 88, 20005. arXiv:0804.3827.

56. Sewell, G.I. Note on the Relativistic Thermodynamics of Moving Bodies. J. Phys. 2010, 43, 485001. arXiv:1010.2045.

57. Yuen, C.K. Lorentz Transformations of Thermodynamic Quantities. Am. J. Phys. 1970, 38, 246. 
58. Nakamura, T.K. Three Views of a Secret in Relativistic Thermodynamics. Prog. Theor. Phys. 2012, 128, 463-475. arXiv:0812.3725.

59. De Parga, G.A.; López-Carrera, B. Redefined Relativistic Thermodynamics based on the Nakamura Formalism. Physica 2009, 388, 4345-4356.

60. Becattini, F. Thermodynamic Equilibrium in Relativity: Four-Temperature, Killing Vectors and Lie Derivatives. arXiv 2016, arXiv:1606.06605.

61. Hilbert, S.; Hänggi, P.; Dunkel, J. Thermodynamical Laws in Isolated Systems. Phys. Rev. 2014, 90, 062116.

62. Poisson, E. The Motion of Point Particles in Curved Spacetime. Living Rev. Relativ. 2004, 7, 6. arXiv:gr-qc/0306052.

63. See [64] for the case of an ideal gas of non-interacting particles.

64. Tuckerman, M. The Ideal Gas. Microcanonical Ensemble Treatment, Lectures in Statistical Mechanics 2003. Available online: www.nyu.edu/classes/tuckerman/stat.mech/lectures/lecture_\{-\}6/ node2.htlm (accessed on 20 February 2003).

65. This is a different problem from how to describe equilibrium in general relativity [66], where there are physical tidal degrees of freedom of the gravitational field, and the equivalence principle forbids the existence of global inertial frames.

66. Bergmann, P. Generalized Statistical Mechanics. Phys. Rev. 1951, 84, 1026.

67. Dunkel, J.; Hänggi, P. Relativistic Brownian Motion. Phys. Rep. 2009, 471, 1-73.

68. Gourgoulhon, E. An Introduction to Relativistic Hydrodynamics. In Stellar Fluid Dynamics and Numerical Simulations: From the Sun to Neutron Stars; EAS Publication Series; Rieutord, M., Dubrulle, B., Eds.; Cambridge University Press: Cambridge, UK, 2006; Volume 21, p. 43. arXiv:gr-qc/0603009.

69. Eyink, G.L.; Drivas, T.D. Cascades and Dissipative Anomalies in Relativistic Fluid Turbolence. arXiv 2017, arXiv:1704.03541.

70. Thyagaraja, A.; McClements, K.G. Plasma Physics in NonInertial Frames. Phys. Plasma 2009, 16, 092506.

71. Apostol, M. Non-Inertial Electromagnetic Effects in Matter: Gyromagnetic Effect. Solid State Commun. 2012, 152, 1567-1571.

72. Biscari, P.; Cercignani, C. Stress and Heat Flux in Non-Inertial Reference Frames. Continuum Mech. Thermodyn. 1997, 9, 1-11.

73. Hernandez, J.; Kovtun, P. Relativistic Magnetohydrodynamics. arXiv 2017, arXiv:1703.08757.

74. The flat Minkowski four-metric is $\eta_{\mu v}=\epsilon(+---)$ with $\epsilon= \pm(\epsilon=+$ is the particle physics convention, $\epsilon=-$ is the general relativity one).

75. Bondi, H. Assumption and Myth in Physical Theory; Cambridge University Press: Cambridge, UK, 1967.

76. Lusanna, L. The Rest-Frame Instant Form of Metric Gravity. Gen. Rel. Grav. 2001, 33, $1579-1696$. arXiv:gr-qc/0101048.

77. Lusanna, L.; Villani, M. Hamiltonian Expression of Curvature Tensors in the York Canonical Basis: I) Riemann Tensor and Ricci Scalars. Int. J. Geom. Methods Mod. Phys. 2014, 11, 1450052. arXiv:1401.1370.

78. Lusanna, L.; Villani, M. Hamiltonian Expression of Curvature Tensors in the York Canonical Basis: II) Weyl Tensor, Weyl Scalars, Weyl Eigenvalues and the Problem of the Observables of the Gravitational Field. Int. J. Geom. Methods Mod. Phys. 2014, 11, 1450053. arXiv:1401.1375.

79. Alba, D.; Lusanna, L. The Einstein-Maxwell-Particle System in the York Canonical Basis of ADM Tetrad Gravity: I) The Equations of Motion in Arbitrary Schwinger Time Gauges. Can. J. Phys. 2012, 90, 1017-1076. arXiv:0907.4087.

80. Alba, D.; Lusanna, L. The Einstein-Maxwell-Particle System in the York Canonical Basis of ADM Tetrad Gravity: II) The Weak Field Approximation in the 3-Orthogonal Gauges and Hamiltonian Post-Minkowskian Gravity: The N-Body Problem and Gravitational Waves with Asymptotic Background. Can. J. Phys. 2012, 90, 1077-1130. arXiv:1003.5143.

81. Alba, D.; Lusanna, L. The Einstein-Maxwell-Particle System in the York Canonical Basis of ADM Tetrad Gravity: III) The Post-Minkowskian N-Body Problem, its Post-Newtonian Limit in Non-Harmonic 3-Orthogonal Gauges and Dark Matter as an Inertial Effect. Can. J. Phys. 2012, 90, 1131-1216. arXiv:1009.1794.

82. Lusanna, L. Non-Inertial Frames in Special and General Relativity. In Gravity: Where do We Stand?; Peron, R., Colpi, M., Gorini, V., Moschella, U., Eds.; Springer: Berlin/Heidelberg, Germany, 2016. arXiv:1310.4465.

83. Lusanna, L. Canonical ADM Tetrad Gravity: From Metrological Inertial Gauge Variables to Dynamical Tidal Dirac Observables. Int. J. Geom. Methods Mod. Phys. 2015, 12, 1530001. arXiv:1401.1375. 
84. The four-vectors $z_{r}^{\mu}(\tau, \vec{\sigma})$ are tangent to $\Sigma_{\tau}$. The unit normal $l^{\mu}(\tau, \vec{\sigma})$ to the three-manifold $\Sigma_{\tau}$ is proportional to $\epsilon_{\alpha \beta \gamma}^{\mu}\left[z_{1}^{\alpha} z_{2}^{\beta} z_{3}^{\gamma}\right](\tau, \vec{\sigma})$. The evolution four-vector is $z_{\tau}^{\mu}(\tau, \vec{\sigma})=\left[N l^{\mu}+n^{r} z_{r}^{\mu}\right](\tau, \vec{\sigma})$, where $N(\tau, \vec{\sigma})=$ $1+n(\tau, \vec{\sigma})=\epsilon\left[z_{\tau}^{\mu} l_{\mu}\right](\tau, \vec{\sigma})$ and $n_{r}(\tau, \vec{\sigma})=-\epsilon g_{\tau r}(\tau, \vec{\sigma})=\left[{ }^{3} g_{r s} n^{s}\right](\tau, \vec{\sigma})$ are the lapse and shift functions (the names used for these functions in general relativity). We also have $\left|\operatorname{det}^{4} g\right|=(1+n) \sqrt{\gamma} ; \sqrt{\gamma}=\sqrt{\operatorname{det}^{3} g}$ with ${ }^{3} g_{r s}=-\epsilon^{4} g_{r s}$ of positive signature.

85. The admissible nice foliations have [25,26]: (i) a lapse function positive in every point, so as to avoid the coordinate singularity of the Fermi coordinates; (ii) $\epsilon^{4} g_{\tau \tau}\left(\tau, \sigma^{r}\right)>0$, so as to avoid the coordinate singularity of the rotating disk; (iii) a positive-definite three-metric ${ }^{3} g_{r s}\left(\tau, \sigma^{u}\right)=-\epsilon^{4} g_{r s}\left(\tau, \sigma^{u}\right)$ with three positive eigenvalues. In [25] there is the explicit form of the functions $z^{\mu}\left(\tau, \sigma^{r}\right)$ of the nice foliations for pure differential rotations (Equation (2.14)) and pure linear acceleration (Equation (2.13)).

86. $T_{\perp \perp}=l_{\mu} l_{v} T^{\mu v}=(1+n)^{2} T^{\tau \tau}, T_{\perp r}=l_{\mu} z_{r v} T^{\mu v}=-(1+n) h_{r s}\left(T^{\tau \tau} n^{s}+T^{\tau s}\right), T_{r s}=z_{r \mu} z_{s v} T^{\mu v}=$ $n_{r} n_{s} T^{\tau \tau}+\left(n_{r} h_{s u}+n_{s} h_{r u}\right) T^{\tau u}+h_{r u} h_{s v} T^{u v}$. In inertial frames, $T_{\perp \perp}=T^{\tau \tau}$ and $T_{\perp r}=\delta_{r s} T^{\tau s}$.

87. Only the configurations of the isolated system having them finite and with the total four-momentum time-like are physically relevant.

88. $\vec{z} / M c=\vec{x}_{N W}(0)$ are the Cauchy data for the Newton-Wigner three-position. Even if the measure $d^{3} z d^{3} h$ is Lorentz invariant, $\vec{z}$ is a non-covariant quantity.

89. The three collective variables $\tilde{x}^{\mu}(\tau), Y^{\mu}(\tau)$ and $R^{\mu}(\tau)$ are well-defined functions of $\tau, \vec{z}, \vec{h}, M, \vec{S}$. All of them tend to the Newtonian center of mass for $c \rightarrow \infty$. Only the Fokker-Pryce center of inertia $Y^{\mu}(\tau)$ is a four-vector with a well-defined world-line. All of the pseudo-world-lines of the other two collective variables fill a non-covariance Möller world-tube of radius $|\vec{S}| / M c$ as discussed in [31].

90. Its expression is $Y^{\mu}(\tau)=Y^{\mu}(0)+h^{\mu} \tau$ with $Y^{\mu}(0)=\left(\sqrt{1+\vec{h}^{2}} \frac{\vec{h} \cdot \vec{z}}{M c} ; \frac{\vec{z}}{M c}+\frac{\vec{h} \cdot \vec{z}}{M c} \vec{h}+\frac{\vec{S} \times \vec{h}}{M c\left(1+\sqrt{1+\vec{h}^{2}}\right)}\right)$.

91. The rest frame conditions $\mathcal{P}^{r} \approx 0$ imply $\vec{q}_{+} \approx \vec{R}_{+} \approx \vec{y}_{+}$, where $\vec{q}_{+}$is the internal canonical three-center of mass (the internal Newton-Wigner position), $\vec{y}_{+}$is the internal Fokker-Pryce three-center of inertia and $\vec{R}_{+}$ is the internal $\mathrm{M}$ ller three-center of energy. As a consequence, there is a unique internal three-center of mass.

92. In the non-relativistic limit, one has $\vec{x}_{i}(\tau) \rightarrow_{c \rightarrow \infty} \vec{x}_{(n)}(t)+(-)^{i+1} \frac{m_{i+1}}{m} \vec{r}_{(n)}(t)=\vec{x}_{(n) i}(t)$, where $\vec{x}_{(n) i}(t)$ are the Newton trajectories $\left(\vec{x}_{(n)}\right.$ and $\vec{r}_{(n)}$ are the Newton center of mass and relative variable, respectively, and $\left.m_{3}=m_{1}\right)$.

93. Instead the rest spin is always $\vec{S}=\sum_{i} \vec{\eta}_{i} \times \vec{\kappa}_{i}$ being in an instant form of dynamics.

94. The expressions in the inertial rest frame are recovered in the flat limit with $T_{F}^{\mu v}=\left.T^{\mu v}\right|_{z_{W}^{\rho}}$.

95. As in [34], the volume is non-dynamical: one considers only the motions of the isolated system of particles contained in it and not dynamical elastic reflections at its boundaries.

96. See Appendix B of [35] for the Galilei generators of N scalar particles in Newtonian gravity and for the ADM Poincaré generators in the Post-Minkowskian approximation [79-81] of Einstein general relativity.

97. Its form is not known in closed form for $m \neq 0$ (for $m=0$, see [98]).

98. Matolcsi, T.; Kristóf, J.; Székely, M. On the Momentum Distribution of Molecules of an Ideal Gas. Publ. Appl. Anal. 1996, 7,1.

99. As said in Section 2.1 the internal energy $M_{N} c=M_{N}\left(\vec{\eta}_{i}, \vec{\kappa}_{i}\right) c$ should be re-expressed in terms of the relative variables after imposing the gauge-fixing $\overrightarrow{\mathcal{K}}_{N} \approx 0$ for the elimination of the internal center of mass: $M_{N} c=$ $\hat{M}_{N}\left(\vec{\rho}_{a}, \vec{\pi}_{a}\right)$. Then, a Jacobian $J\left(\vec{\rho}_{a}, \vec{\pi}_{a}\right)$ appears: it is defined by $\delta^{3}\left(\frac{\overrightarrow{\mathcal{K}}_{N}}{M_{N} c}\right)=J\left(\vec{\rho}_{a}, \vec{\pi}_{a}\right) \delta^{3}\left(\vec{\eta}-\vec{\eta}_{+}\left(\vec{\rho}_{a}, \vec{\pi}_{a}\right)\right)$.

100. The natural volume $V$ would be a spherical box centered on the Fokker-Pryce center of inertia in the Wigner three-space $\left(\left|\vec{\eta}_{i}(\tau)\right| \leq R\right)$ identified by a characteristic function $\chi(V)=\prod_{i} \theta\left(R-\left|\vec{\eta}_{i}(\tau)\right|\right)$. Since the internal center of mass is eliminated, the characteristic function depends only on the relative variables, namely $\chi(V)=\prod_{a} \theta\left(2 R-\left|\vec{\rho}_{a}\right|\right)$.

101. By definition, one has $\int \prod_{i=1}^{N} d^{3} x_{i} d^{3} p_{i} \rho\left(\vec{x}_{1}, \vec{p}_{1}, \ldots, \vec{x}_{N}, \vec{p}_{N}, t\right)=1$.

102. The hypothesis of molecular chaos is needed for its evaluation. Moreover, the distribution function must change slowly over distances and times of the order of the characteristic interaction lengths and durations.

103. Israel, W.; Steward, J.M. Transient Relativistic Thermodynamics and Kinetic Theory. Ann. Phys. 1979, 118, 341-372.

104. Kremer, G.M. On the Kinetic Theory of Relativistic Gases. Continuum Mech. Thermodyn. 1997, 9, $13-21$.

105. Boisseau, B.; Van Leeuwen, W.A. Relativistic Boltzmann Theory in D+1 Spacetime Dimensions. Ann. Phys. 1989, 195, 376-419. 
106. Cercignani, C.; Kreimer, G.M. The Relativistic Boltzmann Equation: Theory and Applications; Birkhäuser: Basel, Switzerland, 2002.

107. Strain, R.M. Global Newtonian Limit for the Relativistic Boltzmann Equation near Vacuum. SIAM J. Math. Anal. 2010, 42, 1568-1601.

108. Strain, R.M. Coordinates in the Relativistic Boltzmann Theory. Kinet. Relat. Model. 2011, 4, 345-359. arXiv:1011.5093.

109. See also [9], where it is shown that if we consider a gas of charged massive particles interacting with external electro-magnetic and gravitational fields, the Hamilton equations of motion of a particle are $\frac{d x^{\mu}(\lambda)}{d \lambda}=p^{\mu}(\lambda)$, $\frac{d p^{\mu}(\lambda)}{d \lambda}=e F^{\mu}{ }_{v}(x(\lambda)) p^{v}(\lambda)-\Gamma_{\alpha \beta}^{\mu}(x(\lambda)) p^{\alpha}(\lambda) p^{\beta}(\lambda)=F^{\mu}(x(\lambda), p(\lambda))(\lambda$ is an affine parameter; self-forces are not considered). Therefore, if $\hat{L}=p^{\mu} \frac{\partial}{\partial x^{\mu}}+F^{\mu} \frac{\partial}{\partial p^{\mu}}$ is the associated Liouville operator, the relativistic

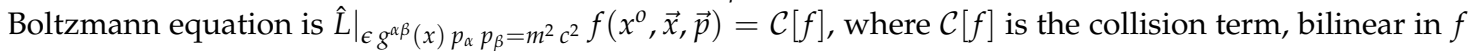
for two-body collisions. At equilibrium (no collisions), the distribution function $f_{e q}\left(x^{0}, \vec{x}, \vec{p}\right)$ satisfies this equation with $\mathcal{C}\left[f_{e q}\right]=0$ and $\partial_{x^{\circ}} f_{e q}=0$.

110. Van Kampen, N.G. Lorentz-Invariance of the Distribution in Phase Space. Physica 1969, 43, $244-262$.

111. Debbasch, F. Equilibrium Distribution Function of a Relativistic Dilute Perfect Gas. Physica 2007, 387, 2443-2454.

112. Debbasch, F.; Rivet, J.P.; van Leeuwen, W.A. Invariance of the Relativistic One-Particle Distribution Function. Physica 2001, 301, 181-195.

113. Chacón-Acosta, G.; Dagdug, L.; Morales-Técotl, H.A. Manifestly Covariant Jüttner Distribution and Equipartition Theorem. Phys. Rev. 2010, 81, 021126. arXiv:0910.1625.

114. Treumann, R.A.; Nakamura, R.; Baumjohann, W. Relativistic Transformation of Phase-Space Distributions. Ann. Geophys. 2011, 29, 1259-1265. arXiv:1105.2120.

115. Cubero, D.; Casado-Pascual, J.; Dunkel, J.; Talkner, P.; Hänggi, P. Thermal Equilibrium and Statistical Thermometers in Special Relativity. Phys. Rev. Lett. 2007, 99, 170601. arXiv:0705.3328.

116. Jüttner, F. Das Maxwellsche Gesetz der Geschwindigkeitsverteilung in der Relativtheorie. Ann. Phys. 1911, 34, 856-882. (In German)

117. Jüttner, F. Die Dynamik eines bewegten Gases in der Relativtheorie. Ann. Phys. 1911, 35, 145-161. (In German)

118. Jüttner, F. Die relativistische Quantentheorie des idealen Gases. Z. Phys. 1928, 47, 542-566. (In German)

119. The four-vector $\beta^{\mu}=\frac{1}{k_{B} T} U^{\mu}$, with $U^{\mu}$ a unit four-vector equal to $(1 ; 0)$ in the rest frame, is a Killing vector; the limit $N, V \rightarrow \infty$ with $N / V=$ const. is assumed.

120. For isolated relativistic systems with short-range interactions, one expects that the four-vector $\beta^{\mu}$ has the form $\beta^{\mu}=\frac{1}{k_{B} T} h^{\mu}$ with $h^{\mu}=P^{\mu} / \sqrt{\epsilon P^{2}}$.

121. In Appendix $A$ of [35] are the Poincaré generators for $N$ charged scalar particles with the Coulomb plus Darwin potentials of [29], while in Appendix B is their expression for N scalar particles in the post-Minkowskian tetrad gravity of [79-81].

122. We assume that $\rho\left(\vec{\eta}_{1}(\tau), . ., \vec{\kappa}_{N}(\tau), \tau\right)$ is symmetric in the $N$ particles as in [1].

123. For instance, there are discussions going on whether the standard definition of relativistic thermodynamics given in [103] is acceptable. See, for instance, [124-126] for a proposal to modify the first member of the Gibbs relation $d e=T d s+\mu d n$ by adding a dependence on the momentum density to the variation $d e$ of the local rest frame energy density.

124. Ván, P. Kinetic Equilibrium and Relativistic Thermodynamics. EPJ Web Conf. 2011, 13, 07004. arXiv:1102.0323.

125. Ván, P. Internal Energy in Dissipative Relativistic Fluids. J. Mech. Mater. Struct. 2008, 3, 1161-1169. arXiv:0712.1437.

126. Ván, P.; Bíró, T.S. Relativistic Hydrodynamics-Causality and Stability. EPJ Spec. Top. 2008, 155, $201-212$. arXiv:0704.2039.

127. Hirano, T.; van der Kolk, N.; Bilandzic, A. Hydrodynamics and Flows. In The Physics of the Quark-Gluon Plasma; Sarkar, S., Satz, H., Sinha, B., Eds.; Springer: Berlin/Heidelberg, Germany, 2010.

128. Romatschke, P. Relativistic Viscous Fluid Dynamics and Non-Equilibrium Entropy. Class. Quantum Gravity 2009, 27, 025006. arXiv:0906.4787.

129. Romatschke, P. New Developments in Relativistic Viscous Hydrodynamics. Int. J. Mod. Phys. 2010, 19, 1-53. arXiv:0902.3663.

130. Csernai, L.P. Introduction to Relativistic Heavy Ion Collisions; Wiley: New York, NY, USA, 2008. 
131. Andersson, N.; Comer, G.L. Relativistic Fluid Dynamics: Physics for Many Different Scales. Living Rev. Relativ. 2007, 10, 1. arXiv:gr-qc/0605010.

132. Andersson, N.; Lopez-Monsalvo, C. A Consistent First-Order Model for Relativistic Heat Flow. Class. Quantum Gravity 2011, 28, 195023. arXiv:1107.0165.

133. Andersson, N.; Comer, G.L. Variational Multi-Fluid Dynamics and Causal Heat Conductivity. Proc. R. Soc. 2010, 466, 1373-1387. arXiv:0908.1707.

134. López-Monsalvo, C. Covariant Thermodynamics and Relativity. Ph.D. Thesis, University of Southampton, Southampton, UK, 6 July 2011. arXiv:1107.1005.

135. Carter, B. Covariant Theory of Conductivity in Ideal Fluids or Solid Media. In Lecture Notes in Mathematics 1385; Springer: Berlin/Heidelberg, Germany, 1989.

136. Maartens, R. Causal Thermodynamics in Relativity. In Proceedings of the Lectures at the Hanno Rund Workshop on Relativity and Thermodynamics; Natal University: Durban, South Africa, 1996. arXiv:astro-ph/9609119.

137. Lusanna, L.; Nowak-Szczepaniak, D. The Rest-Frame Instant Form of Relativistic Perfect Fluids with Equation of State $\rho=\rho(\eta, s)$ and of Nondissipative Elastic Materials. Int. J. Mod. Phys. 2000, 15, 4943-5016.

138. Denicol, G.S.; Koide, T.; Rischke, D.H. Dissipative Relativistic Fluid Dynamics: A New Way to Derive the Equations of Motion from Kinetic Theory. Phys. Rev. Lett. 2010, 105, 162501. arXiv:1004.5013.

139. Betz, B.; Denicol, G.S.; Koide, T.; Molnár, E.; Niemi, H.; Rischke, D.H. Second order Dissipative Fluid Dynamics from Kinetic Theory. EPJ Web Conf. 2011, 13, 07005. arXiv:1012.5772.

140. Betz, B.; Henkel, D.; Rischke, D.H. Complete Second-Order Dissipative Fluid Dynamics. J. Phys. 2009, $36,064029$.

141. Monsalvo, C.S.; Andersson, N. Thermal Dynamics in General Relativity. Proc R. Soc. Lond. 2010, 467, $738-759$. arXiv:1006.2978.

142. Chen, X. Causal Relativistic Fluid Dynamics. Class. Quantum Gravity 2011, 28, 175003. arXiv:1107.0319

143. Priou, D. Comparison between Variational and Traditional Approaches to Relativistic Thermodynamics of Dissipative Fluids. Phys. Rev. 1991, 43, 1223.

144. Brown, J.D. Action Functionals for Relativistic Perfect Fluids. Class. Quantum Gravity 1993, 10, $1579-1606$.

145. It has no connection with the $T^{\mu v}$ of Section 2.

146. Takamoto, M.; Inutsuka, S. The Relativistic Kinetic Dispersion Relation: Comparison of the Relativistic Bhatnagar-Gross-Krook Model and Grad's 14-Moment Expansion. Phys. A Stat. Mech. Appl. 2010, 389, 4580-4603. arXiv:1006.2663

147. Alba, D.; Lusanna, L. Generalized Eulerian Coordinates for Relativistic Fluids: Hamiltonian Rest-Frame Instant Form, Relative Variables, Rotational Kinematics. Int. J. Mod. Phys. 2004, 19, 3025. arXiv:hep-th/0209032.

148. Alba, D.; Lusanna, L. Dust in the York Canonical Basis of ADM Tetrad Gravity: The Problem of Vorticity. Int. J. Geom. Methods Mod. Phys. 2015, 12, 1550076. arXiv:1106.0403

149. Anco, S.C.; Dar, A. Conservation Laws of Inviscid Non-Isentropic Compressible Fluid Flow in $n>1$ Spatial Dimension. Proc. R. Soc. Lond. A 2010, doi:10.1098/rspa.2009.0579. arXiv:0911.0882.

150. In the $3+1$ formalism of Section 2 , it is connected to the skew congruence of time-like observers with four-velocity $z_{\tau}^{\mu}(\tau, \vec{\sigma})$. See [148] for the properties of the two congruences of observers in the case of dust: In general, the dust four-velocity is different from both of these four-velocities.

(C) 2017 by the author. Licensee MDPI, Basel, Switzerland. This article is an open access article distributed under the terms and conditions of the Creative Commons Attribution (CC BY) license (http://creativecommons.org/licenses/by/4.0/). 